\title{
QoS-Aware and Energy-Efficient Resource Management in OFDMA Femtocells
}

\author{
Long Bao Le, Senior Member, IEEE, Dusit Niyato, Member, IEEE, Ekram Hossain, Senior Member, IEEE, Dong \\ In Kim, Senior Member, IEEE, and Dinh Thai Hoang
}

\begin{abstract}
We consider the joint resource allocation and admission control problem for Orthogonal Frequency-Division Multiple Access (OFDMA)-based femtocell networks. We assume that Macrocell User Equipments (MUEs) can establish connections with Femtocell Base Stations (FBSs) to mitigate the excessive cross-tier interference and achieve better throughput. A crosslayer design model is considered where multiband opportunistic scheduling at the Medium Access Control (MAC) layer and admission control at the network layer working at different time-scales are assumed. We assume that both MUEs and Femtocell User Equipments (FUEs) have minimum average rate constraints, which depend on their geographical locations and their application requirements. In addition, blocking probability constraints are imposed on each FUE so that the connections from MUEs only result in controllable performance degradation for FUEs. We present an optimal design for the admission control problem by using the theory of Semi-Markov Decision Process (SMDP). Moreover, we devise a novel distributed femtocell power adaptation algorithm, which converges to the Nash equilibrium of a corresponding power adaptation game. This power adaptation algorithm reduces energy consumption for femtocells while still maintaining individual cell throughput by adapting the FBS power to the traffic load in the network. Finally, numerical results are presented to demonstrate the desirable operation of the optimal admission control solution, the significant performance gain of the proposed hybrid access strategy with respect to the closed access counterpart, and the great power saving gain achieved by the proposed power adaptation algorithm.
\end{abstract}

Index Terms-Femtocell network, admission control, Markov decision process, blocking probability, channel assignment.

\section{INTRODUCTION}

$\mathbf{S}$ MALL cell deployment and inter-cell interference mitigation have been recognized as the key techniques to enhance the capacity of cellular wireless networks [1]. Due to

Manuscript received January 29, 2012; revised May 27 and August 02, 2012; accepted September 30, 2012. The associate editor coordinating the review of this paper and approving it for publication is M. C. Gursoy.

This work was supported in part by the Natural Sciences and Engineering Research Council of Canada (NSERC) under the Strategic Grant Program and the MKE, Korea, under the ITRC support program supervised by the NIPA (NIPA-2012-(H0301-12-1005)). This paper was presented in part at IEEE International Conference on Communications (IEEE ICC'2012), Ottawa, Ontario, Canada.

L. B. Le is with the Institut National de la Recherche Scientifique - Énergie, Matériaux et Télécommunications (INRS-EMT), Université du Québec, Montréal, Québec, Canada (e-mail: long.le@emt.inrs.ca).

D. Niyato and D. T. Hoang are with Nanyang Technological University, Singapore (e-mail: \{dniyato, thdinh $\} @$ ntu.edu.sg).

E. Hossain is with University of Manitoba, Winnipeg, MB, Canada (e-mail: Ekram.Hossain@ad.umanitoba.ca).

D. I. Kim is with Sungkyunkwan University (SKKU), Suwon, Korea (email: dikim@skku.ac.kr).

Digital Object Identifier 10.1109/TWC.2012.120412.120141. minimal installation and operation costs, emerging femtocells have been shown to be a viable technical solution for broadband wireless access in indoor environments [2]. However, since femtocells may operate on the same frequency spectrum as macrocells, mitigation of cross-tier interference is a very significant research issue.

To enable spectrum sharing between macrocells and femtocells in Orthogonal Frequency-Division Multiple Access (OFDMA)-based two-tier networks, one of the three access modes, namely, open, closed and hybrid access, can be employed [3]-[7]. In the open access mode, the MUEs are allowed to connect to either their own Macrocell BSs (MBS) or FBSs. In contrast, in the closed access mode, only certain users (subscribers) belonging to the so-called Closed Subscriber Group are allowed to connect to each FBS. The hybrid access mode balances the strengths and weaknesses of the closed and open access modes. In a typical hybrid access mode for OFDMA-based two-tier network, the FUEs can use all available subchannels when there is no connected MUE. However, limited spectrum access at each femtocell is granted for MUEs which wish to establish connections. An efficient admission control policy is required in this case to coordinate spectrum sharing and admission control for both types of users, which should strike a balance between achieving high spectrum utilization and protecting QoS requirements for FUEs. In this paper, we develop such a spectrum sharing and admission control mechanism for OFDMA-based two-tier networks.

There have been some recent works on resource allocation and performance analysis of OFDMA-based femtocell networks. In [5], a static frequency assignment scheme based on fractional frequency reuse was proposed considering the handoff, coverage, and interference aspects of femtocell networks. In [8], a randomized frequency allocation strategy called FALOHA was proposed and its area spectral-efficiency was analyzed for OFDMA-based femtocell networks by using stochastic geometry. Research studies in [9] suggested that open access can provide a throughput gain of more than $300 \%$ for CDMA-based femtocell networks whereas the throughput performance of open and closed access modes for OFDMA femtocells depends on user density. Moreover, the admission control and handoff problem for femtocell networks was studied by using simulations [4].

In [10], a hierarchical resource allocation framework for OFDMA-based femtocell networks was proposed that includes three control loops, namely, maximum power setting for FUEs, 
target SINR assignment for FUEs, and instantaneous power control to achieve these target SINR values. In [11], a distributed power control algorithm was proposed for femtocells to maximize the sum-rate achieved by FUEs. In [12], to design resource allocation algorithms, a game model was used, the equilibria of which were shown to be fair and efficient. In [13], a frequency scheduling algorithm based on spectrum sensing was proposed for coexistence of MUEs and FUEs. In [14], distributed admission control and spectrum allocation algorithms were developed using reinforcement learning, which are, however, unable to provide performance guarantees for users of both network tiers.

There are existing works in the literature that investigated the admission control problem based on Markov Decision Process (MDP) and also the power control problem for traditional one-tier CDMA wireless networks [16], [17], [21]. To the best of our knowledge, the problem of optimal admission control with quality-of-service (QoS) guarantee in multi-tier wireless cellular networks, which is addressed in this paper, has not been well investigated in the literature. The contributions of this paper can be summarized as follows:

- We develop a mathematical model for joint resource allocation and admission control design for dynamic spectrum sharing in OFDMA-based two-tier femtocell networks. This model considers the QoS requirements of MUEs and FUEs in terms of average rates and blocking probabilities in presence of both co-tier and cross-tier interferences.

- We devise a cross-layer radio resource management framework for femtocells with a multiband proportionalfair scheduling scheme at the MAC layer and an admission control scheme at the network layer. Then, the optimal admission control solution is obtained by using the theory of Semi-Markov Decision Process (SMDP).

- For energy-efficient resource management, we propose a novel distributed femtocell power adaptation algorithm by using game theory. We prove that the proposed femtocell power adaptation algorithm converges to the Nash equilibrium (NE) of the game.

- We demonstrate the efficacy of the proposed admission control scheme as well as the significant power saving gains of the proposed power adaptation algorithm via numerical studies.

The rest of this paper is organized as follows. In Section II, we present the system model. The cross-layer resource allocation and admission control framework is presented in Section III. We present the distributed femtocell power adaptation algorithm in Section IV. Numerical results are presented in Section V which is followed by conclusion in Section VI. A summary of key notations is presented in Table I.

\section{SySTEM MODEL}

We consider the downlink of a two-tier OFDMA-based wireless network that employs Frequency-Division Duplexing (FDD). There are $N$ subchannels shared by MUEs and FUEs for downlink communications ${ }^{1}$. It is assumed that there are

\footnotetext{
${ }^{1}$ The structure of cross-tier interference in the uplink is quite different from that in the downlink. Analysis of the uplink scenario is left for our future work.
}

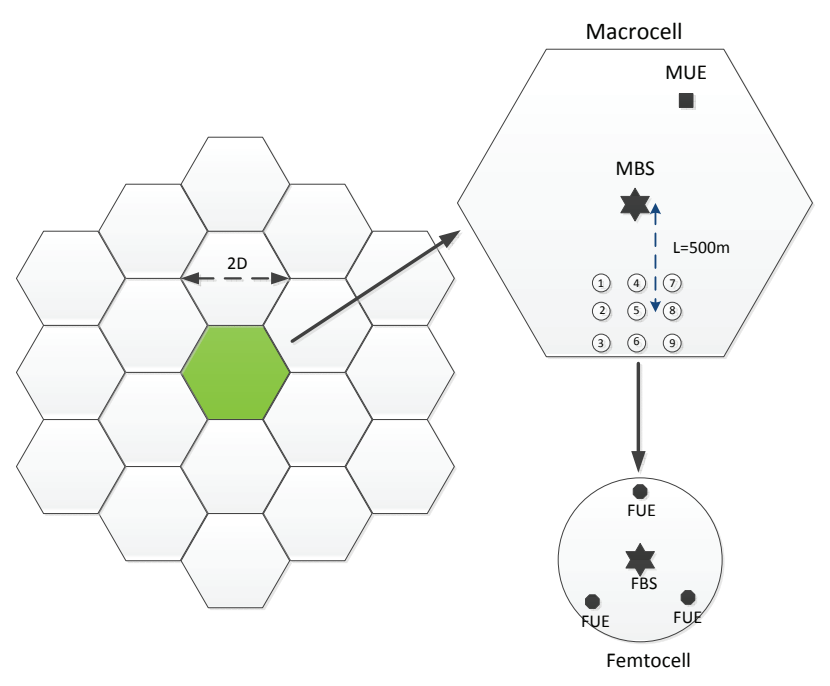

Fig. 1. Two-tier femtocell network.

$J$ femtocells sharing these $N$ subchannels with $I$ macrocells. We further assume that a hybrid access policy is employed where MUEs can connect to a nearby FBS. This can happen when MUEs suffer from undue cross-tier interference or they can achieve better rates if connected with a nearby FBS. The system model under consideration is illustrated in Fig. 1.

We assume that each subchannel can be assigned to at most one FUE or MUE connecting to any FBS. Furthermore, we assume a full spatial reuse where all subchannels are utilized at each FBS. In addition, subchannels are assumed to be allocated to macrocells in such a way that interference among macrocells is properly controlled ${ }^{2}$. We consider the scenario where users of both network tiers demand some minimum average rates which are determined by their underlying applications and locations in the cell. In fact, location-dependent QoS constraints can be imposed to balance user throughput and fairness. For instance, cell-edge users may require a minimum rate that is smaller than that of cell-center users so that the total cell throughput is not severely compromised. We assume that there are $C_{1}$ classes of FUEs and $C_{2}$ classes of MUEs connecting to any FBS. In addition, it is assumed that class- $c$ MUEs and FUEs require their total average rates to be at least $R_{\min }^{(m c)}$ and $R_{\min }^{(f c)}$, respectively.

To illustrate these QoS constraints, let us assume that longterm signal attenuation depends on the distance between users and a BS symmetrically. Note however that this assumption can be relaxed as long as detailed path-loss information in each cell is available. The area around each FBS can be divided into circular areas and users running the same application (e.g., voice or video) in each circular area can be imposed the same minimum average rate requirement. Each such minimum rate is mapped to one user class. We depict this QoS modeling in Fig. 2 where the femtocell area is divided into cell-edge and cell-center regions. In addition, there is a circular region where MUEs inside that region would request to establish connections with the underlying FBS (i.e., they switch their

\footnotetext{
${ }^{2}$ Examples of subchannel allocation schemes include fractional frequency reuse and partial frequency reuse [15].
} 
TABLE I

SUMMARY OF KEY NOTATIONS

\begin{tabular}{|c|c|}
\hline Notation & Physical meaning \\
\hline $\bar{I}$ & Number of macrocells \\
\hline$J$ & Number of femtocells \\
\hline$N$ & Number of subchannels \\
\hline$W$ & Bandwidth of one subchannel \\
\hline$C_{1}, C_{2}$ & Number of FUE/MUE service classes \\
\hline$R_{\min }^{(f c)}, R_{\min }^{(m c)}$ & Minumum required rate for class- $c$ FUEs/MUEs \\
\hline$g_{i j k}^{(f c)}, g_{i j k}^{(f c)}$ & Channel gain from FBS/MBS $j$ to class- $c$ user $k$ in femtocell $i$ \\
\hline$L\left(d_{i j k}\right)$ & Path-loss over distance $d_{i j k}$ \\
\hline$\lambda_{i c}^{(f)}, \lambda_{i c}^{(m)}$ & Arrival rate of class- $c$ FUEs/MUEs in femtocell $i$ \\
\hline$\mu_{i c}^{(f)}, \mu_{i c}^{(m)}$ & Service rate of class- $c$ FUEs/MUEs in femtocell $i$ \\
\hline$p_{i}, p_{0}$ & Transmission power on each subchannel from FBS/MBS $i$ \\
\hline$\beta_{i}$ & Transmission power ratio for femtocell $i$ \\
\hline$P_{\mathrm{FBS}}^{\max }, P_{\mathrm{MBS}}^{\max }$ & Maximum transmission power of FBS/MBS \\
\hline$\Gamma_{i k}^{(f c)}, \Gamma_{i k}^{(m c)}$ & SINR of class- $c$ FUE/MUE $k$ in femtocell $i$ \\
\hline $\bar{\Gamma}_{i k}^{2 k}(f c), \bar{\Gamma}_{i k}^{2 m c)}$ & Average SINR of class- $c$ FUE/MUE $k$ in femtocell $i$ \\
\hline$U_{i c}^{(f)}, U_{i c}^{(m)}$ & Set of class- $c$ FUEs/MUEs in femtocell $i$ \\
\hline$u_{i c}^{(f)}, u_{i c}^{(m)}$ & Number of class- $c$ FUEs/MUEs in femtocell $i$ \\
\hline$\Delta_{i c}^{(f)}, \Delta_{i c}^{(m)}$ & Set of subchannels allocated for class- $c$ FUEs/MUEs in femtocell $i$ \\
\hline$s_{i c}^{(f)}, s_{i c}^{(m)}$ & Number of subchannels allocated for class- $c$ FUEs/MUEs in femtocell $i$ \\
\hline$\underline{s}_{i c}^{(f)}, \underline{s}_{i c}^{(m)}$ & Required number of subchannels for class- $c$ FUEs/MUEs in femtocell $i$ \\
\hline$y_{i k}^{(f c)}, y_{i k}^{(m c)}$ & Equal $\Gamma_{i k}^{(f c)} / \bar{\Gamma}_{i k}^{(f c)}$ and $\Gamma_{i k}^{(m c)} / \bar{\Gamma}_{i k}^{(m c)}$, respectively \\
\hline$f_{y_{i k}^{(f c)}}(x), F_{y_{i k}(f c)}(x)$ & Probability density function and probability distribution function for $y_{i k}^{(f c)}$ \\
\hline$f_{y_{i k}^{(m c)}}(x), F_{y_{i k}^{(m c)}}(x)$ & Probability density function and probability distribution function for $y_{i k}^{(m c)}$ \\
\hline$r_{i k}^{(f c)}, r_{i k}^{(m c)}$ & Average rate achieved by class- $c$ FUE/MUE $k$ in femtocell $i$ \\
\hline$\underline{r}_{i k}^{(f c)}, \underline{r}_{i k}^{(m c)}$ & Minimum average rate achieved by class- $c$ FUE/MUE $k$ in femtocell $i$ \\
\hline$B_{i c}^{(f)}, B_{i c}^{(m)}$ & Blocking probability for class- $c$ FUEs/MUEs in femtocell $i$ \\
\hline$P_{b}^{(f c)}, P_{b}^{(m c)}$ & Target blocking probability for class- $c$ FUEs/MUEs in femtocell $i$ \\
\hline$a_{i c}^{(f)}, a_{i c}^{(m)}$ & Control action of class- $c$ FUEs/MUEs in femtocell $i$ \\
\hline$A_{x}$ & Admissible action space for system state $x$ \\
\hline$\tau_{x}(a)$ & Expected time until the next decision epoch when action $a$ is taken \\
\hline$z_{x a}$ & Rate of choosing action $a$ in state $x$ \\
\hline$w_{i c}^{(m)}, w_{i c}^{(f)}$ & Weighting factors defining the cost function of the MDP formulation \\
\hline$T_{i}, T$ & Throughput of femtocell $i$ and total throughput \\
\hline$\sigma$ & Standard deviation of shadowing \\
\hline$\delta$ & Power scaling factor \\
\hline$P_{i}, F_{i}()$. & Power and payoff function of femtocell $i$ \\
\hline$p_{x y}(a)$ & Transition probability from state $x$ to state $y$ given action $a$ be taken \\
\hline
\end{tabular}

connections to the underlying FBS) ${ }^{3}$.

We assume that the distance from any user connecting with FBS $i$ to other FBS/MBS $j$ can be well approximated by the distance from FBS $i$ to FBS/MBS $j$. This assumption would be valid because the size of a typical femtocell would be much smaller than the distance between the BSs of either tier. Let $g_{i j k}^{(f c)}$ and $g_{i j k}^{(m c)}$ be the channel gains on a particular subchannel from FBS $j$ and MBS $j$, respectively, to a particular class- $c$ user $k$ of either tier who is associated with femtocell $i$. For brevity, the subchannel index is omitted in these notations. Channel gains are modeled considering pathloss, shadowing, and Rayleigh fading. Specifically, we can write $g_{i j k}^{(x c)}$ ( $x$ stands for $f$ or $m$ ) as $g_{i j k}^{(x c)} \triangleq L\left(d_{i j k}\right) 10^{\eta / 10} \omega$, where $L\left(d_{i j k}\right)$ represents the path-loss over the corresponding distance $d_{i j k}, \eta$ is a Gaussian-distributed random variable with zero mean and standard deviation $\sigma$, and $\omega$ represents the short-term Rayleigh fading gain. In addition, we assume that

\footnotetext{
${ }^{3}$ MUEs typically achieve better average SINRs and higher transmission rates when they enter this neighborhood and switch their connections to the underlying FBS.
}

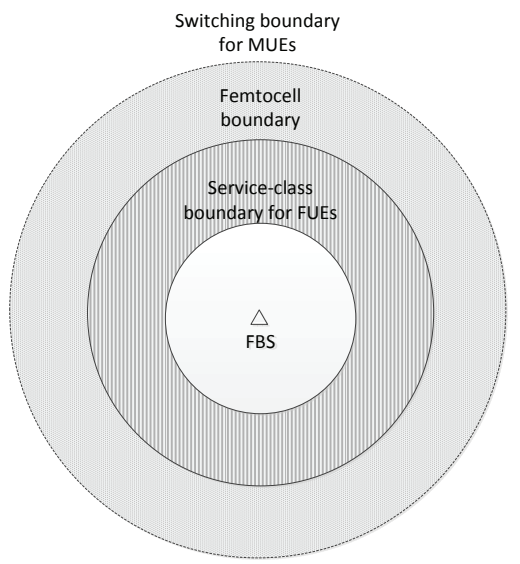

Fig. 2. Location-dependent QoS classes and switching boundary for MUEs.

connection requests of class- $c$ FUEs and MUEs in femtocell $i$, which are assumed to follow Poisson processes, arrive with rate $\lambda_{i c}^{(f)}$ and $\lambda_{i c}^{(m)}$, respectively. The connection duration is 


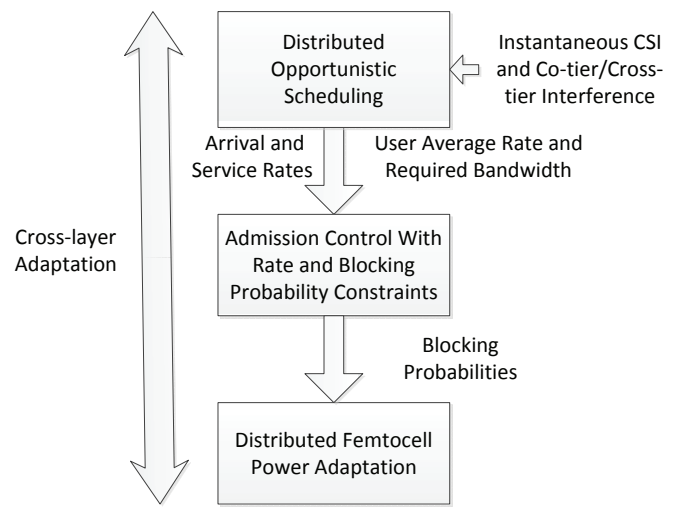

Fig. 3. Cross-layer resource management model with time-scale separation.

assumed to be exponentially distributed with mean duration of $1 / \mu_{i c}^{(f)}$ and $1 / \mu_{i c}^{(m)}$ for class-c FUEs and MUEs, respectively.

\section{CRoss-Layer Resource Allocation And ADMISSION CONTROL FRAMEWORK}

We consider a cross-layer joint resource allocation and admission control framework for femtocells with the following network functionalities. A distributed opportunistic scheduling algorithm is assumed to be implemented at each FBS to exploit the multiuser diversity at a small time-scale. Depending on the channel dynamics observed by users in two tiers, the size of a scheduling time slot is assumed to be designed accordingly. In contrast, the admission controller operates at a larger timescale, which captures the system dynamics due to user arrivals and departures. Also, we design a distributed power adaptation algorithm that adapts the transmission power of FBSs to the heterogeneous traffic distribution over the network for QoSaware and energy-efficient spectrum sharing between the two network tiers.

The cross-layer model under consideration is illustrated in Fig. 3. It is worth noting that we consider a dynamic network model where users come and leave the network over time. Therefore, traditional resource allocation algorithms developed for a static and snapshot network model with fixed network topology (i.e., fixed number of users with known locations) cannot be applied to our setting.

We assume that the MBSs have maximum transmission power of $P_{\mathrm{MBS}}^{\max }$ while the maximum allowable power of FBSs is $P_{\mathrm{FBS}}^{\max }$ which is determined from outage probability constraints for MUEs and will be described in Section III-C. For simplicity, we assume that the MBSs and FBSs perform uniform power allocation over the subchannels and FBS $j$ uses $0 \leq \beta_{j} \leq 1$ fraction of its maximum power while the MBSs use their maximum power for downlink communications ${ }^{4}$. These femtocell transmission power ratios $\beta_{j}$ will be employed by the distributed power adaptation algorithm to be presented in Section IV. Therefore, the transmission power on any subchannel for users connecting to FBS $j$ is $p_{j}=\beta_{j} P_{\mathrm{FBS}}^{\max } / N$ and the transmission power from any MBS on one subchannel is $p_{0}=P_{\mathrm{MBS}}^{\max } / N$. Given the power

\footnotetext{
${ }^{4}$ We do not consider resource allocation for the macrocell tier in this paper. However, the proposed algorithms would be able to adapt to the potential dynamic operations of the macrocells.
}

allocation for MBSs and FBSs, we present an opportunistic scheduling algorithm and analyze its throughput performance in the following.

\section{A. Multi-band Opportunistic Scheduling}

The signal-to-interference-plus-noise ratio (SINR) achieved by class- $c$ FUE $k$ associated with femtocell $i$ on a particular subchannel can be written as

$$
\begin{aligned}
\Gamma_{i k}^{(f c)} & =\frac{p_{i} g_{i i k}^{(f c)}}{\sum_{j=1, j \neq i}^{J} g_{i j k}^{(f c)} p_{j}+\sum_{j=1}^{I} g_{i j k}^{(m c)} p_{0}+N_{0}} \\
& =\frac{p_{i} g_{i i k}^{(f c)}}{\sum_{j=1, j \neq i}^{J} g_{i j k}^{(f c)} p_{j}+I N_{i k}^{(f c)}}
\end{aligned}
$$

where $N_{0}$ denotes the noise power and recall that $g_{i j k}^{(f c)}$ and $g_{i j k}^{(m c)}$ are the channel gains from FBS $j$ and MBS $j$ to class- $c$ FUE $k$ in femtocell $i$, respectively. The first and second terms in the denominator of (1) represent the total interference due to other FBSs and MBSs, respectively. In addition, $I N_{i k}^{(f c)}=$ $\sum_{j=1}^{I} g_{i j k}^{(m c)} p_{0}+N_{0}$ denotes the total interference from MBSs and the noise power. Similarly, the SINR achieved by class- $c$ MUE $k$ connecting with FBS $i$ can be written as

$$
\begin{aligned}
\Gamma_{i k}^{(m c)} & =\frac{p_{i} g_{i i k}^{(m c)}}{\sum_{j=1, j \neq i}^{J} g_{i j k}^{(f c)} p_{j}+\sum_{j=1, j \neq j_{i}}^{I} g_{i j k}^{(m c)} p_{0}+N_{0}} \\
& =\frac{p_{i} g_{i i k}^{(m c)}}{\sum_{j=1, j \neq i}^{J} g_{i j k}^{(f c)} p_{j}+I N_{i k}^{(m c)}}
\end{aligned}
$$

where $I N_{i k}^{(m c)}=\sum_{j=1, j \neq j_{i}}^{I} g_{i j k}^{(m c)} p_{0}+N_{0}$ and $j_{i}$ is the nearest MBS of femtocell $i$. Here, we assume that subchannels allocated to MUEs connected with FBS $i$ are not assigned to other MUEs connected with the nearest MBS of femtocell $i$. Hence, in the second term in the denominator of (2) we exclude the interference from this MBS in calculating the total noise and interference power. For brevity, we omit the subchannel index in the SINR notations $\Gamma_{i k}^{(f c)}$ and $\Gamma_{i k}^{(m c)}$. We assume that the SINR-proportional fair opportunistic scheduling algorithm [20], [22] is employed on each subchannel ${ }^{5}$. In particular, time is divided into equal-size time slots and scheduling decisions are made in each time slot for all subchannels. Because the time slot interval is very small (e.g., typically few milliseconds) compared to the user dwelling time, the opportunistic scheduling algorithm operates over a small timescale. Note that a particular user of either tier can be scheduled to transmit on multiple subchannels in each time slot.

Let $U_{i c}^{(m)}$ and $U_{i c}^{(f)}$ denote the sets of class-c MUEs and FUEs in femtocell $i$ and their cardinalities are denoted as $u_{i c}^{(m)}=\left|U_{i c}^{(m)}\right|$ and $u_{i c}^{(f)}=\left|U_{i c}^{(f)}\right|$, respectively. In addition, let $\Delta_{i c}^{(f)}$ and $\Delta_{i c}^{(m)}$ be the sets of subchannels allocated for class- $c$ FUEs and MUEs in femtocell $i$, respectively. We assume that these sets are determined to meet the average rate requirements and they are only updated when the numbers of active users

\footnotetext{
${ }^{5}$ Consideration of the proportional fair scheduling in the cross-layer model would be quite natural given that it has been adopted in practical wireless standards [20], [22]. Moreover, the framework considered in this paper can be readily extended to other scheduling schemes.
} 
$u_{i c}^{(m)}$ and $u_{i c}^{(f)}$ change. The SINR-proportional fair opportunistic scheduling algorithm operates on a particular subchannel $v \in \Delta_{i c}^{(f)}$ as follows. FBS $i$ chooses FUE $k^{*}$ that achieves the largest ratio $\Gamma_{i k}^{(f c)}(t) / \bar{\Gamma}_{i k}^{(f c)}$ for transmission in time slot $t$ on this subchannel, i.e., $k^{*} \triangleq \operatorname{argmax}_{k \in U_{i c}^{(f)}} \Gamma_{i k}^{(f c)}(t) / \bar{\Gamma}_{i k}^{(f c)}$ where recall that $\Gamma_{i k}^{(f c)}(t)$ and $\bar{\Gamma}_{i k}^{(f c)}$ are the instantaneous and average SINR of FUE $k$ on the underlying subchannel, respectively. Similar operations are applied for subchannels in $\Delta_{i c}^{(m)}$ to schedule MUEs in the sets $U_{i c}^{(m)}$.

Now, we determine the average rate achieved by a particular FUE $k \in U_{i c}^{(f)}$ on one particular subchannel. Toward this end, we need to describe the probability distribution of $\Gamma_{i k}^{(f c)}(t) / \bar{\Gamma}_{i k}^{(f c)}$. It was shown in [19], [20] that the composite variability of $g_{i j k}^{(f c)}$ due to Rayleigh fading and lognormal shadowing can be approximated by a single lognormal distribution. In addition, it is well-known that a sum of lognormally-distributed random variables can be represented by a single lognormal distribution [23], [32]. Also, the ratio of two lognormally-distributed random variables is lognormally distributed. Therefore, $y_{i k}^{(f c)}=\Gamma_{i k}^{(f c)}(t) / \bar{\Gamma}_{i k}^{(f c)}$ can be approximated by a lognormally-distributed random variable whose mean and standard deviation can be calculated as in [20]. Then, the average rate achieved by FUE $k \in U_{i c}^{(f)}$ on a particular subchannel can be written as follows ${ }^{6}$ :

$$
\begin{aligned}
r_{i k}^{(f c)}=W \int_{0}^{\infty} \log \left(1+y_{i k}^{(f c)} \bar{\Gamma}_{i k}^{(f c)}\right) \\
\times\left\{\prod_{j \in U_{i, c}^{(f)}, j \neq k} F_{y_{i j}^{(f c)}}(x)\right\} f_{y_{i k}^{(f c)}}(x) d x
\end{aligned}
$$

where $W$ is the bandwidth of one subchannel; $f_{y_{i k}^{(f c)}}(x)$ and $F_{y_{i k}^{(f c)}}(x)$ represent the probability density function and probability distribution function of $y_{i k}^{(f c)}$, respectively. Similarly, the average rate achieved by MUE $k \in U_{i c}^{(m)}$ on a particular subchannel can be written as follows:

$$
\begin{aligned}
r_{i k}^{(m c)}=W & \int_{0}^{\infty} \log \left(1+y_{i k}^{(m c)} \bar{\Gamma}_{i k}^{(m c)}\right) \\
& \times\left\{\prod_{j \in U_{i c}^{(m)}, j \neq k} F_{y_{i j}^{(m c)}}(x)\right\} f_{y_{i k}^{(m c)}}(x) d x
\end{aligned}
$$

where $y_{i k}^{(m c)}=\Gamma_{i k}^{(m c)}(t) / \bar{\Gamma}_{i k}^{(m c)} ; f_{y_{i k}^{(m c)}}(x)$ and $F_{y_{i k}^{(m c)}}(x)$ represent the probability density function and probability distribution function of $y_{i k}^{(m c)}$, respectively.

\section{B. QoS Constraints}

It can be verified that the average rate $r_{i k}^{(f c)}$ calculated in (3) decreases with the path-loss $L\left(d_{i i k}\right)$ for given power allocation and channel gain parameters. Moreover, $r_{i k}^{(f c)}$ decreases with the number of class-c FUEs $u_{i c}^{(f)}$ because the proportional-fair

\footnotetext{
${ }^{6}$ Assuming that the long-term channel gains on different subchannels are the same and the typical user dwelling time is much larger than a scheduling time slot, the average rates achieved by a particular user on different subchannels are the same.
}

scheduling allows equal long-term time-shares among users [18], [20] and each subchannel in $\Delta_{i c}^{(f)}$ is shared by $u_{i c}^{(f)}$ FUEs. Recall that class- $c$ MUEs and FUEs require their total average rates to be at least $R_{\min }^{(m c)}$ and $R_{\min }^{(f c)}$, respectively. To determine the minimum number of subchannels to meet the minimum rate requirement, we consider the worst user with minimum $L\left(d_{i i k}\right)$ (or maximum $d_{i i k}$ ) for each service class $c$ in femtocell $i$. That is, the femtocell $i$ is located at the edge of the corresponding service class for symmetric path-loss.

Let $\underline{r}_{i}^{(f c)}$ and $\underline{r}_{i}^{(m c)}$ be the minimum rates achieved by any class- $c$ FUE and MUE, respectively. These minimum rates can be calculated by using (3) and (4) for the corresponding worst users. Let $s_{i c}^{(f)}=\left|\Delta_{i c}^{(f)}\right|$ and $s_{i c}^{(m)}=\left|\Delta_{i c}^{(m)}\right|$ be the numbers of subchannels allocated for class- $c$ FUEs and MUEs in femtocell $i$, respectively. To maintain the rate requirements for FUEs and MUEs of each service class, we impose the following constraints ${ }^{7}$ :

$$
\begin{array}{r}
R_{\min }^{(f c)} \leq s_{i c}^{(f)} \times \underline{r}_{i}^{(f c)}\left(u_{i c}^{(f)}\right) \\
R_{\min }^{(m c)} \leq s_{i c}^{(m)} \times \underline{r}_{i}^{(m c)}\left(u_{i c}^{(m)}\right)
\end{array}
$$

where we explicitly describe the dependence of $\underline{r}_{i}^{(f c)}$ and $\underline{r}_{i}^{(m c)}$ on $u_{i c}^{(f)}$ and $u_{i c}^{(m)}$, respectively. These constraints ensure that the minimum rate requirements are satisfied by any class$c$ user. From these inequalities, we require that the numbers of subchannels allocated for class- $c$ MUE and FUE satisfy

$$
\begin{gathered}
s_{i c}^{(f)} \geq\left\lceil\frac{R_{\min }^{(f c)}}{\underline{r}_{i}^{(f c)}\left(u_{i c}^{(f)}\right)}\right\rceil \triangleq \underline{s}_{i c}^{(f)}\left(u_{i c}^{(f)}\right) \\
s_{i c}^{(m)} \geq\left\lceil\frac{R_{\min }^{(m c)}}{\underline{r}_{i}^{(m c)}\left(u_{i c}^{(m)}\right)}\right\rceil \triangleq \underline{s}_{i c}^{(m)}\left(u_{i c}^{(m)}\right) .
\end{gathered}
$$

Therefore, the rate constraints in (5) and (6) hold if the following constraints for femtocell $i$ are satisfied:

$$
N \geq \sum_{c=1}^{C_{1}} \underline{s}_{i c}^{(f)}\left(u_{i c}^{(f)}\right)+\sum_{c=1}^{C_{2}} \underline{s}_{i c}^{(m)}\left(u_{i c}^{(m)}\right), \quad \forall i=1,2, \ldots, J .
$$

This constraint means that the number of available subchannels should be large enough to support the required minimum rates for all service classes. This constraint will be used for admission control design. Since the MUEs degrade the performance of the FUEs connecting to the same FBS, the FUEs must be satisfactorily protected. Toward this end, we assume that a class- $c$ FUE has the maximum tolerable blocking probability of $P_{b}^{(f c)}$. Let $B_{i c}^{(f)}$ denote the blocking probability of class- $c$ FUEs in femtocell $i$. Then, the blocking probability constraints can be written as follows:

$$
B_{i c}^{(f)} \leq P_{b}^{(f c)}, \quad \forall i, c .
$$

The admission control and channel assignment should be performed in such a way that they can satisfy the channel and blocking probability constraints in (9) and (10), respectively.

\footnotetext{
${ }^{7}$ Since the average rates achieved by any user on different subchannels are the same, only the number of subchannels allocated to a particular service class impacts the achievable average rates of its users. Note, however, that we have exploited the channel dynamics to enhance the user throughput through employing the proportional fair scheduling.
} 


\section{Maximum Power Constraints Under Closed and Hybrid} Access

Let $d_{\mathrm{MU}}$ be the radius of the circular area centered around each FBS where MUEs inside this region will connect with the corresponding FBS under the proposed hybrid access scheme. Also, let $d_{\mathrm{F}}$ be the radius of the coverage area of any femtocell. We consider both closed and hybrid access strategies and derive the corresponding maximum power constraints for FBSs so that MUEs which are connected with MBSs and close to FBSs are protected from excessive cross-tier interference. Specifically, it is required that the transmission powers of FBSs must be smaller than some maximum value so that outage probability of MUEs is smaller than a desirable value. Toward this end, let us consider a particular MUE $k$ that connects with a nearby MBS and FBS $i$ is its closest FBS. Let $h_{i k}^{(m)}$ be the channel gain between the considered MUE $k$ and its connecting MBS. Then, we can express the SINR of this MUE $k$ as

$$
\begin{aligned}
\gamma_{i k}^{(m)} & =\frac{p_{0} h_{i k}^{(m)}}{\sum_{j=1}^{J} g_{i j k}^{(f c)} p_{j}+\sum_{j=1, j \neq j_{i}}^{I} g_{i j k}^{(m c)} p_{0}+N_{0}} \\
& =\frac{p_{0} h_{i k}^{(m)}}{\sum_{j=1}^{J} g_{i j k}^{(f c)} p_{j}+I N_{i k}^{(m c)}} .
\end{aligned}
$$

Note that all femtocells including femtocell $i$ create cross-tier interference for the considered MUE $k$. In addition, MUEs are not allowed to connect with nearby FBSs under the closed access. To calculate the maximum allowable power for FBSs under the closed access, we impose the outage probability constraints for the worst MUE $k$ whose distance from FBS $i$ is exactly $d_{F}$. Let $\gamma_{0}$ be the target SINR of the considered MUE $k$ then the outage probability constraint can be expressed as

$$
\operatorname{Pr}\left\{\gamma_{i k}^{(m)}\left(d_{F}\right)<\gamma_{0}\right\}<P_{\circ}^{(m)}
$$

where $\gamma_{i k}^{(m)}\left(d_{\mathrm{F}}\right)$ denotes the SINR of the MUE whose distance to the nearest FBS is $d_{\mathrm{F}}$ and $P_{\mathrm{o}}^{(m)}$ represents the target outage probability. The LHS of this inequality denotes the outage probability for the considered MUE. Recall that $\gamma_{i k}^{(m)}$ can be modeled as a lognormally distributed random variable with mean and standard variation (in $\mathrm{dB}$ ) of $\mu_{m}$ and $\eta_{m}$, respectively, which can be calculated as in [20]. The outage probability in the left hand side of (12) can be expressed as [20]

$$
\operatorname{Pr}\left\{\gamma_{i k}^{(m)}\left(d_{F}\right)<\gamma_{0}\right\}=1-\frac{1}{2} \operatorname{erfc}\left(\frac{10 \log _{10} \gamma_{0}-\mu_{m}}{\sqrt{2} \eta_{m}}\right)
$$

where $\operatorname{erfc}(x)$ denotes the complementary error function, defined as $\operatorname{erfc}(x)=\frac{2}{\sqrt{\pi}} \int_{x}^{\infty} \exp \left(-y^{2}\right) d y$. Then, we assume that the transmission power of any FBS is constrained by a common maximum value $P_{\mathrm{FBS}}^{\max }$ so that outage probability constraints in (12) are satisfied for neighboring MUEs of all femtocells. This kind of outage probability constraints has been considered in several recent works [27], [28]. For the proposed hybrid access, any MUE whose distance from the nearest FBS is less than $d_{\mathrm{MU}}$ will connect with the FBS. Therefore, the outage probability constraints in this case can be expressed as

$$
\operatorname{Pr}\left\{\gamma_{i k}^{(m)}\left(d_{\mathrm{MU}}\right)<\gamma_{0}\right\}<P_{\mathrm{o}}^{(m)} .
$$

The outage probability in (14) can be calculated as in (13) with the corresponding mean and standard deviation values. And the maximum transmission power $P_{\mathrm{FBS}}^{\max }$ of all FBS under the hybrid access can be calculated so that the outage probability constraints in (14) are satisfied. The maximum transmission power $P_{\mathrm{FBS}}^{\max }$ under either access scheme is assumed to be estimated by FBSs during a network startup phase.

\section{SMDP-Based Distributed Admission Control in Femtocells}

Given the considered physical and network layer models, admission control is performed at the network layer considering user dynamics at the call level. It turns out that admission control can be performed separately in each cell, which enables distributed implementation. We will present and analyze the performance of an optimal admission control scheme for a particular femtocell $i$. In fact, we can formulate the admission control problem as an SMDP [33].

We proceed by describing the state and action spaces for the underlying SMDP. The decision epochs of the underlying SMDP are arrival and departure instants of either FUE or MUE in the considered femtocell $i$. We define a general system state at decision epoch $t$ as follows:

$$
x(t) \triangleq\left[u_{i 1}^{(f)}(t), \ldots, u_{i C_{1}}^{(f)}(t), u_{i 1}^{(m)}(t), \ldots, u_{i C_{2}}^{(m)}(t)\right]
$$

where recall that $u_{i c}^{(f)}(t)$ and $u_{i c}^{(m)}(t)$ are the numbers of class$c$ FUEs and MUEs at decision epoch $t$, respectively. Then, the state space $X$ is defined as follows:

$$
X \triangleq\{x: \text { constraint (9) holds }\} .
$$

At each decision epoch when the system changes its state, an admission control action is determined for the next decision epoch. In fact, an admission control action is only taken for a newly arriving user of either type. At a departure instant of any connection, state transition occurs and no action is needed. We define a general action $a$ at decision epoch $t$ as

$$
a(t) \triangleq\left[a_{i 1}^{(f)}(t), \ldots, a_{i C_{1}}^{(f)}(t), a_{i 1}^{(m)}(t), \ldots, a_{i C_{2}}^{(m)}(t)\right]
$$

where $a_{i c}^{(f)}(t)$ and $a_{i c}^{(m)}(t)$ denote the admission control action when an arrival occurs for class-c FUEs and MUEs in femtocell $i$, respectively, which are defined as follows:

$$
a_{i c}^{(f)}= \begin{cases}1, & \text { if a newly arriving FUE is admitted } \\ 0, & \text { otherwise }\end{cases}
$$

$a_{i c}^{(m)}= \begin{cases}1, & \text { if a newly arriving MUE is admitted } \\ 0, & \text { otherwise. }\end{cases}$

The action state space can be defined as $A=$ $\left\{a: a \in\{0,1\}^{C_{1}+C_{2}}\right\}$. Given these system and action state spaces, we can determine the transition probabilities for the underlying embedded Markov chains based on which the optimal solution can be obtained (see Appendix A). Let $B_{i c}^{(f)}$ and $B_{i c}^{(m)}$ be the blocking probabilities for class- $c$ FUEs and 
MUEs, respectively, which can be calculated from the SMDPbased admission control solution presented in Appendix A. Then, we can calculate the achievable call throughput of femtocell $i$ as follows:

$$
T_{i}=\sum_{c=1}^{C_{1}}\left(1-B_{i c}^{(f)}\right) \lambda_{i c}^{(f)}+\sum_{c=1}^{C_{2}}\left(1-B_{i c}^{(m)}\right) \lambda_{i c}^{(m)}
$$

where we have taken blocked arrivals into consideration. Finally, the total network throughput achieved by all femtocells can be calculated as $T=\sum_{i=1}^{f} T_{i}$. The admission control framework developed in this section will be used in the design of a femtocell power adaptation algorithm in the next section.

\section{Distributed Power Adaptation Algorithm}

High transmission power at an FBS may severely degrade the performance of other highly-loaded femtocells and macrocells because of excessive inter-cell and cross-tier interference. To address this issue, we develop an efficient power adaption mechanism by using game theory ${ }^{8}$. The proposed power adaptation algorithm aims to adapt transmission powers of FBSs to spatial traffic load over the network [31] ${ }^{9}$. Therefore, it is only activated when the traffic load described by user arrival rates changes.

\section{Femtocell Power Adaptation Game (FPAG):}

- Players: J FBSs.

- Strategies: Each FBS $i$ can select its total transmission power $P_{i} \in\left[0, P_{\mathrm{FBS}}^{\max }\right]$ (the transmission power ratio $\beta_{i}$ for FBS $i$ is in $[0,1])$ to maximize its payoff.

- Payoffs: $F_{i}\left(P_{i}, P_{-i}\right)$ for each FBS $i$ is $F_{i}\left(P_{i}, P_{-i}\right)=$ $-P_{i}$, where $P_{-i} \triangleq\left[P_{1}, \ldots, P_{i-1}, P_{i+1}, \ldots, P_{J}\right]$ denotes a vector containing transmission powers of other FBSs excluding FBS $i$.

It is assumed that all FBSs are required to satisfy the channel and blocking probability constraints in (9) and (10), respectively, while maximizing their payoffs. In addition, we require that each FBS $i$ that wishes to reduce its transmission power must satisfy the following blocking probability constraints for their MUEs

$$
B_{i c}^{(m)} \leq P_{b}^{(m c)}, 1 \leq c \leq C_{2}
$$

given that these constraints are satisfied when the maximum power $P_{\mathrm{FBS}}^{\max }$ is used. Here $P_{b}^{(m c)} \mathrm{s}$ are some predetermined values. These additional constraints ensure that satisfactory performance in terms of blocking probabilities is achieved for users of both network tiers while the payoffs of all the FBSs are maximized.

Remark 1: We assume that only FBSs participate in the power adaptation game. In general, this formulation can be readily extended to the scenario where MBSs are also players of the game. However, MBSs are typically inter-connected by high-speed links, which enable them to achieve certain global

\footnotetext{
${ }^{8}$ In general, femtocell owners would be interested in maximizing their own benefits while maintaining required $\mathrm{QoS}$ for their users. Therefore, use of non-cooperative game theory in designing the power adaptation algorithm for femtocells arises quite naturally.

${ }^{9}$ The related research topics including design of FPGA, power circuitry, and sleep mode strategies are other aspects of cross-layer design in attaining the energy efficiency, which are out of the scope of this paper.
}

objectives. Investigation of power control issues for MBSs are, therefore, beyond the scope of this paper.

One important concept in game theory is the Nash equilibrium (NE), which is defined in the following.

Definition 1: A transmission power vector $\mathbf{P}^{*}$ is called the NE of the FPAG if for each FBS $i, F_{i}\left(P_{i}^{*}, P_{-i}^{*}\right) \geq F_{i}\left(P_{i}, P_{-i}^{*}\right)$, $\forall P_{i} \in\left[0, P_{\mathrm{FBS}}^{\mathrm{max}}\right]$.

In the following, we develop a femtocell power adaptation algorithm that converges to the NE of the FPAG. In particular, FBSs, whose required blocking probability constraints are still maintained, decrease their transmission powers by a factor $\delta<$ 1 iteratively. Toward this end, we present some preliminary results that characterize the system behavior under these power scaling operations.

Proposition 1: Given the channel gains for all users, we have the following properties:

1) For any FUE and MUE, whose FBS scales down power, the SINR given in (1) and (2) decreases;

2) For any FUE and MUE, whose FBS does not scale down power, the SINR given in (1) and (2) increases.

Proof: To prove the first property of this proposition, let us consider the SINR on any subchannel achieved by a class- $c$ FUEs associated with FBS $i$ which scales down power by a factor $\delta$. Let $\Omega$ be the set of FBSs which scale down their transmission powers excluding FBS $i$ in the current iteration. Then, we can rewrite the SINR of FUE $i$ as follows:

$$
\begin{aligned}
\Gamma_{i k}^{(f c)}= & \frac{\delta p_{i}^{(p)} g_{i i k}^{(f c)}}{\sum_{j \in \Omega} g_{i j k}^{(f c)} \delta p_{j}^{(p)}+\sum_{j \notin \Omega} g_{i j k}^{(f c)} p_{j}^{(p)}+I N_{i k}^{(f c)}} \\
& =\frac{p_{i}^{(p)} g_{i i k}^{(f c)}}{\sum_{j \in \Omega} g_{i j k}^{(f c)} p_{j}^{(p)}+\sum_{j \notin \Omega} g_{i j k}^{(f c)} \frac{p_{j}^{(p)}}{\delta}+\frac{I N_{i k}^{(f c)}}{\delta}} \\
& \leq \frac{p_{i}^{(p)} g_{i i k}^{(f c)}}{\sum_{j \in \Omega} g_{i j k}^{(f c)} p_{j}^{(p)}+\sum_{j \notin \Omega} g_{i j k}^{(f c)} p_{j}^{(p)}+I N_{i k}^{(f c)}}
\end{aligned}
$$

where $p_{j}^{(p)}=\beta_{j} P_{\mathrm{FBS}}^{\max } / N$ is the transmission power on any subchannel in femtocell $j$ in the previous iteration. The last inequality in (22) holds because $\delta<1$. It can be observed that the quantity in the right hand side of (22) is indeed the SINR of class- $c$ FUEs in femtocell $i$ in the previous iteration. Similarly, we can prove the decrease of SINR of MUEs. Therefore, the first property stated in the proposition holds.

The second property in the proposition can be proved similarly. In particular, for any FBS which does not scale down power, the received powers of its FUEs remain the same. However, the total received interference can only decrease due to the decreases in transmission powers of some other FBSs.

The results in Proposition 1 describe how the power updates of FBSs impact the SINRs of users in the network. We now state further results on the average rates achieved by the opportunistic scheduling scheme under power adaptation.

Proposition 2: We have the following results for the average rates achieved by the SINR-proportional fair scheduling scheme:

1) For any FUE and MUE whose FBS scales down power, the average rate given in (3) and (4) decreases; 
2) For any FUE and MUE whose FBS does not scale down power, the average rate given in (3) and (4) increases.

Proof: From Proposition 1, for any FUE and MUE whose connecting FBS scales down the transmission power, its average SINR and rate in each scheduled slot decreases. In addition, one important principle of the proportional fair scheduling is that it allows equal long-term time shares among competing users on each subchannel [18], [20]. Therefore, for the fixed number of users of either type (i.e., FUEs or MUEs) in a particular service class, each user is asymptotically scheduled for the same fraction of time. Since the average rate of each user in each scheduled slot decreases with the decreasing FBS transmission power, its long-term average rate decreases. Therefore, the first property in Proposition $\mathbf{2}$ holds. The second property can be proved similarly by using the fact that the average SINR and rate achieved by any FUE or MUE in each scheduled slot increases in this case.

According to (7) and (8), the number of subchannels required to support certain target minimum rates can increase or decrease if the average rates of the corresponding user class decrease or increase, respectively. In turn, the channel requirements for different user classes impact the channel constraint in (9) and the corresponding admission control performance. Because we are interested in the throughput performance at the network layer, the impacts of power adaptation on the admission control performance must be characterized. Let $u_{i}=\left[u_{i 1}^{(f)}, \ldots, u_{i C_{1}}^{(f)}, u_{i 1}^{(m)}, \ldots, u_{i C_{2}}^{(m)}\right]$ be the vector whose elements represent the number of users of different classes and let $\underline{s}_{i}=\left[\underline{s}_{i 1}^{(f)}, \ldots, \underline{s}_{i C_{1}}^{(f)}, \underline{s}_{i 1}^{(m)}, \ldots, \underline{s}_{i C_{2}}^{(m)}\right]$ be the required numbers of subchannels to support the minimum rate requirements in (7) and (8), respectively.

Definition 2: A vector $\underline{s}_{i}$ is said to dominate another vector $\underline{s}_{i}^{\prime}$ written as $\underline{s}_{i} \succ \underline{s}_{i}^{\prime}$ if we have $\underline{s}_{i c}^{(f)} \geq \underline{s}_{i c}^{\prime}(f), c=1, \ldots, C_{1}$, $\underline{s}_{i c}^{(m)} \geq \underline{s}_{i c}^{(m)}, c=1, \ldots, C_{2}$, and there is at least one strict inequality in these inequalities.

We are ready to state an important result that describes the interaction of the physical and network layer performance of the proposed cross-layer design model.

Proposition 3: Consider the performance of the SMDPbased optimal admission control described in Section III.C under two different channel requirement vectors $\underline{s}_{i}$ and $\underline{s}_{i}^{\prime}$ where $\underline{s}_{i} \succ \underline{s}_{i}^{\prime}$. Let $B_{i c}^{(f)}, B_{i c}^{(m)}$ and $B_{i c}^{\prime(f)}, B_{i c}^{\prime(m)}$ be the optimal blocking probabilities that are attained with the two different channel requirement vectors $\underline{s}_{i}$ and $\underline{s}_{i}^{\prime}$, respectively. Suppose that arrival rates are sufficiently high so that $B_{i c}^{(f)}=$ $B_{i c}^{\prime(f)}=P_{b}^{(f c)}, 1 \leq c \leq C_{1}$ under the SMDP-based admission control $^{10}$. Then, we have $B_{i c}^{\prime(m)} \leq B_{i c}^{(m)}$.

Proof: Let us rewrite the cost function $F_{\pi}$ in (29) for a particular stationary policy $\pi$ as

$$
F_{\pi}=\sum_{c=1}^{C_{1}} w_{i c}^{(f)} B_{i c}^{(f)}+\sum_{c=1}^{C_{2}} w_{i c}^{(m)} B_{i c}^{(m)}
$$

where recall that $B_{i c}^{(f)}$ and $B_{i c}^{(m)}$ denote the blocking probabilities of class- $c$ FUEs and MUEs, respectively.

\footnotetext{
${ }^{10} \mathrm{As}$ will be seen in the numerical results, these probability constraints for FUEs are met with equality for sufficiently large arrival rates.
}

Let $\pi^{*}$ denote the optimal policy of the system with the channel requirement vector $\underline{s}_{i}$ and let $B_{i c}^{(f)}$ and $B_{i c}^{(m)}$ be the optimal blocking probabilities achieved by this optimal policy for FUEs and MUEs, respectively. Refer to Appendix A for further details on how to calculate the optimal admission control solution for a given channel requirement vector. Suppose we apply this policy $\pi^{*}$ for another system with the channel requirement vector $\underline{s}_{i}^{\prime}$. Since we assume $\underline{s}_{i} \succ \underline{s}_{i}^{\prime}$, such policy utilization can maintain the subchannel constraint in (9) and the achievable blocking probabilities satisfy $B_{i c}^{\prime \prime}(f)=B_{i c}^{(f)}=P_{b}^{(f c)}$ (the second equality holds due to the assumption of the proposition) and $B_{i c}^{\prime \prime}(m)=B_{i c}^{(m)}$.

Now, let us compare the blocking probabilities $B_{i c}^{\prime \prime}(m)$ obtained above with the blocking probabilities $B_{i c}^{\prime(m)}$ attained by the optimal solution for the system with the channel requirement vector $\underline{s}_{i}^{\prime}$. We must have $B_{i c}^{\prime(m)} \leq B_{i c}^{\prime \prime}(m)$ since $B_{i c}^{\prime(m)}$ is due to the optimal policy for the system with the channel requirement vector $\underline{s}_{i}^{\prime}$. Therefore, we have $B_{i c}^{\prime(m)} \leq$ $B_{i c}^{\prime \prime}(m)=B_{i c}^{(m)}$. This completes the proof of the proposition.

The results in Proposition 2 and Proposition 3 establish the foundation to develop the power adaptation algorithm. Specifically, we can allow lightly-loaded FBSs to reduce their transmission powers, which in turn increases the blocking probabilities of their users as long as the resulting blocking probabilities satisfy the constraints in (10) and (21). The reduction of transmission powers in lightly-loaded FBSs will increase transmission rates for femtocells with high traffic load. This can potentially decrease channel requirement vector $\underline{s}_{i}$ for other highly-loaded femtocell $i$. This can result in an improvement in blocking probabilities and the total throughput in these highly-loaded femtocells.

The proposed power adaptation algorithm is described in details in Algorithm 1. In steps 3-5, each FBS in set $A_{f}$ attempts to reduce its transmission power by a factor $\delta$. Parameter $P_{b}^{(m c)}$ in (21) is introduced to maintain the performance of MUEs connecting with lightly-loaded FBSs. When multiple femtocells scale down their powers at the same time there may be a subset of these femtocells failing to maintain their blocking probability requirements (i.e., constraints (10) and (21)). In steps 7-9 of Algorithm 1, the femtocells which fail to maintain their blocking probability requirements scale up their powers by a factor $1 / \delta$ to do so. There are subtle interactions among femtocells after step 9. Specifically, the power scaleup of femtocells in steps 7-9 may make other femtocells violate their blocking probability requirements. This is because a larger transmission power of any femtocell results in higher interference and therefore lower transmission rates for users in other femtocells. In steps 10-15 of Algorithm 1, any violating femtocell scales up its transmission power to maintain its blocking probability requirement. This is performed until the blocking probability requirements in all femtocells are satisfied.

The proposed algorithm can be implemented in a distributed fashion because each FBS only needs to know the average rates achieved by its FUEs and MUEs of different classes. This can be attained by letting FUEs and MUEs of all classes 


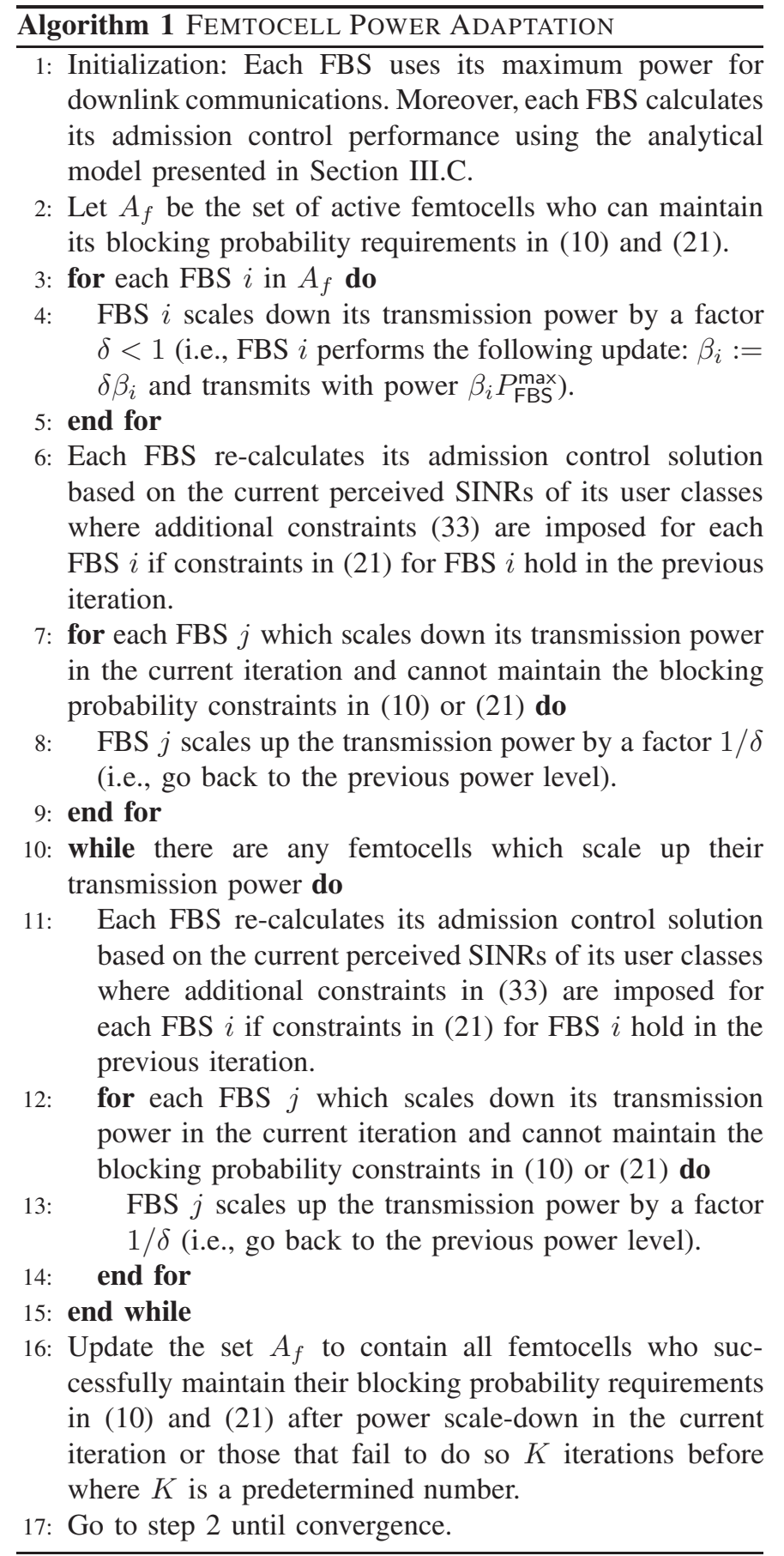

to feedback the estimated mean and standard variation of the lognormally distributed variables representing their SINRs to each FBS $i$, which are used to calculate the average rates and channel requirement vector $\underline{s}_{i}$. The channel requirement vectors are used to calculate the admission control performance for users of both tiers in each femtocell. The convergence of Algorithm 1 is stated in the following theorem.

Theorem 4: We have the following results for Algorithm 1:

1) The blocking probability requirements in (10) and (21) are satisfied at the end of each iteration.

2) Algorithm 1 converges to an equilibrium.

3) If $\delta$ is sufficiently close to 1 then the achieved equilibrium tends to the NE of the FPAG.
Proof: Note that the interference received by any FUEs and MUEs does not increase over iterations; therefore, if a femtocell goes back to its power level in the previous iteration their blocking probability constraints must be satisfied. Also, since the blocking probability requirements of all lightlyloaded femtocells are satisfied in the first iteration and there is a finite number of femtocells, the inner loop corresponding to steps 10-15 of Algorithm 1 must terminate in a finite number of sub-iterations (bounded by the number of femtocells). Therefore, the blocking probability requirements in (10) and (21) for all femtocells are satisfied at the end of each iteration (i.e., after step 15). Hence, we have completed the proof for the first property of this theorem.

We now prove the convergence of Algorithm 1. In each iteration, each femtocell either utilizes the same power level as in the previous iteration or scales down its power by a factor $\delta<1$ in Algorithm 1. Recall that the power adaptation operations performed in Algorithm 1 can maintain the blocking probability constraints (10) and (21) for all femtocells in any iteration (the first property of the theorem). In order to maintain these constraints, the average achieved rates of associated users must be strictly larger than zero (since the blocking probabilities of any user class tend to one if the average achieved rate of that user class tends to zero (i.e., zero service rate)). Since each FUE and MUE receive nonzero total noise and interference power from other MBSs, the transmission power of any FBS must be strictly larger than zero to achieve non-zero average rates for its associated users. This implies that Algorithm 1 must converge to an equilibrium at which no femtocell can scale down its power further.

Finally, we prove that the achieved equilibrium tends to the NE of the FPAG for $\delta$ sufficiently close to 1 by contradiction. Specifically, suppose that the achieved equilibrium is not a NE. Then there must exist an FBS which can increase its payoff or slightly decrease its transmission power while still maintaining the constraints in (10) and (21) given the transmission powers of other FBSs. However, this contradicts to the fact that Algorithm 1 cannot decrease the transmission power of any FBS further at the equilibrium. Therefore, the achieved equilibrium must be close to the NE of the FPAG if $\delta$ is sufficiently close to 1 .

Let $\beta_{i}^{*}, i=1, \ldots, J$ be the femtocell transmission power ratios obtained by Algorithm 1 at convergence. Then, we can calculate the power saving with respect to the maximum allowed transmission power as follows:

$S_{p}=100 \times \frac{J P_{\mathrm{FBS}}^{\max }-\sum_{i=1}^{J} \beta_{i}^{*} P_{\mathrm{FBS}}^{\max }}{J P_{\mathrm{FBS}}^{\max }}=100 \times \frac{J-\sum_{i=1}^{J} \beta_{i}^{*}}{J}$.

One may want to compare the admission control performance at the equilibrium attained by Algorithm 1 with that achieved by using the maximum power. We characterize these results in the following theorem.

Theorem 5: We have the following results for the equilibrium achieved by Algorithm 1.

1) Throughput reduction $\Delta T_{i}$ of a lightly-loaded femtocell $i$, which scales down its transmission power, can be upper-bounded as $\Delta T_{i} \leq \sum_{c=1}^{C_{1}} P_{b}^{(f c)} \lambda_{i c}^{(f)}+$ 
$\sum_{c=1}^{C_{2}} P_{b}^{(m c)} \lambda_{i c}^{(m)}$

2) Let $\underline{s}_{i}$ and $\underline{s}_{i}^{\prime}$ be channel requirement vectors of a particular highly-loaded femtocell $i$ under maximum power and at the equilibrium attained by Algorithm 1. Suppose the arrival rates of the considered femtocell are sufficiently large such that the constraints in (10) are met with equality when the maximum power is used. In addition, assume that we have $\underline{s}_{i}^{\prime} \prec \underline{s}_{i}$. Then, Algorithm 1 results in better throughput for the considered femtocell $i$ compared to that achieved by using the maximum power.

Proof: The first result of this theorem can be proved by noting that a lightly-loaded femtocell $i$ only decreases its transmission power as long as the blocking probability constraints in (10) and (21) are still satisfied. Using the fact that the blocking probabilities for FUEs and MUEs are upperbounded by $P_{b}^{(f c)}$ and $P_{b}^{(m c)}$, respectively, the upper-bound in throughput reduction can be obtained by consulting the throughput formula in (20).

The second result of this theorem can be proved by using the results in Proposition 3. In particular, the blocking probabilities for FUEs and MUEs decrease under Algorithm 1 because we have $\underline{s}_{i}^{\prime} \prec \underline{s}_{i}$. Therefore, the second result follows by using these results and the throughput formula in (20).

Remark 2: The power adaptation operations can be implemented asynchronously without impacting its convergence. In fact, in the arguments employed to prove the convergence of Algorithm 1 we do not impose any restriction on the number of femtocells that scale down transmission powers in any iteration. Therefore, the convergence holds for both synchronous and asynchronous power updates. Moreover, the choice of parameter $\delta$ in Algorithm 1 impacts the tradeoff between convergence speed and the transmission powers at the equilibrium. In particular, fast convergence can be achieved by smaller $\delta$ at the cost of larger transmission powers at the equilibrium.

Remark 3: Let us define $\lambda_{\text {tot }}=\sum_{c=1}^{C_{1}} \lambda_{i c}^{(f)}+\sum_{c=1}^{C_{2}} \lambda_{i c}^{(m)}$. Suppose we choose $P_{b}^{(f c)}=P_{b}^{(m c)}=P_{b}$; then, the throughput loss of a lightly-loaded femtocell $i$ presented in Theorem 5 can be rewritten as $\Delta T_{i} \leq P_{b} \lambda_{\text {tot }}$. Since $\lambda_{\text {tot }}$ is bounded in stable systems we can achieve any desirable throughput loss by choosing $P_{b}$ sufficiently small. This implies that the NE achieved by Algorithm 1 indeed results in better energy efficiency for the femtocell network with improved throughput for highly-loaded femtocells and negligible throughput loss for lightly-loaded femtocells. Therefore, the achieved NE is a desirable operating point.

Remark 4: Although the NE may not be very efficient compared to certain globally optimal solution, choosing NE as an operating point for the femtocells can be justified by the following facts. Firstly, femtocells' owners would be typically selfish and only interested in optimizing their own benefits. Hence, NE would be an appropriate solution concept that enables us to realize this expectation. Secondly, the proposed power adaptation algorithm (i.e., Algorithm 1), which converges to the $\mathrm{NE}$ of the underlying game, can be implemented in a distributed manner. This is very desirable as backhaul links over which signaling information can be ex- changed typically have limited capacity. Thirdly, development of a distributed algorithm to reach a certain globally optimal solution for the considered multi-layer resource allocation and admission control problem seems to be non-tractable. In fact, it has been well-known that the utility maximization problem for multicell OFDMA setting even under the static scenario (i.e., fixed number of users without dynamic user arrival and departure) is NP-hard and very complex to solve up to optimality [29]. Therefore, formulating the power adaption as a game where we can reach the $\mathrm{NE}$ in a distributed manner would be a natural design approach for the femtocell network.

Remark 5: We can calculate the energy efficiency for the proposed hybrid access scheme in terms of energy per bit [24], [25], [26]. Assume that users of each service class transmit data at the required minimum rates, i.e., class- $c$ MUEs and FUEs transmit at rates $R_{\min }^{(m c)}$ and $R_{\min }^{(f c)}$, respectively. Let $\bar{N}_{i}^{(f c)}$ and $\bar{N}_{i}^{(m c)}$ be the average numbers of class-c FUEs and MUEs connected with FBS $i$. Then, we can calculate the average numbers of bits transmitted by FBS $i$ in one second as

$$
S_{i}=\sum_{c=1}^{C_{1}} \bar{N}_{i}^{(f c)} R_{\min }^{(m c)}+\sum_{c=1}^{C_{2}} \bar{N}_{i}^{(m c)} R_{\min }^{(f c)} .
$$

Recall that FBS $i$ transmits at the power level $P_{i}=\beta_{i} P_{\mathrm{FBS}}^{\max }$. Therefore, we can calculate the overall energy efficiency of all femtocells as

$$
\begin{aligned}
E & =\frac{\sum_{i=1}^{J} P_{i}}{\sum_{i=1}^{J} S_{i}} \\
& =\frac{\sum_{i=1}^{J} \beta_{i} P_{\mathrm{FBS}}^{\max }}{\sum_{i=1}^{J}\left(\sum_{c=1}^{C_{1}} \bar{N}_{i}^{(f c)} R_{\min }^{(m c)}+\sum_{c=1}^{C_{2}} \bar{N}_{i}^{(m c)} R_{\min }^{(f c)}\right)} .
\end{aligned}
$$

This implies that we can calculate the energy efficiency if we can calculate $\bar{N}_{i}^{(f c)}$ and $\bar{N}_{i}^{(m c)}$ for a given power usage profile of all femtocells (i.e., $\beta_{i}$ for all femtocells $i=1, \ldots, J$ ) and an admission control policy. Unfortunately, it seems nontractable to find closed-form expressions of $\bar{N}_{i}^{(f c)}$ and $\bar{N}_{i}^{(m c)}$ for the proposed optimal admission control policy. However, we can quantify the performance gain of the proposed power adaptation algorithm (i.e., Algorithm 1) in terms of energy efficiency with respect to the scenario where the maximum transmission power $P_{\mathrm{FBS}}^{\max }$ is used if we can calculate the ratio of $S_{\text {tot }}=\sum_{i=1}^{J}\left(\sum_{c=1}^{C_{1}} \bar{N}_{i}^{(f c)} R_{\min }^{(m c)}+\sum_{c=1}^{C_{2}} \bar{N}_{i}^{(m c)} R_{\min }^{(f c)}\right)$ under these two power usage profiles (i.e., $S_{\text {tot }}^{1} / S_{\text {tot }}^{2}$ where $S_{\text {tot }}^{1}$ and $S_{\text {tot }}^{2}$ denote the values of $S_{\text {tot }}$ under the two power usage profiles, respectively).

We can argue that the values of $S_{\text {tot }}$ are approximately the same under these two power usage profiles. In fact, Algorithm 1 only scales down powers of lightly-loaded femtocells to the extent that the blocking probabilities of all associated MUEs and FUEs are below the predetermined small target blocking probabilities. In addition, any femtocell having higher average service rate compared to other femtocells reduces its transmission power. Since the interference decreases with the transmission power, all femtocells would have roughly the same "service rates" under both power usage profiles. Since the energy efficiency given in (26) is inversely proportional to $S_{\text {tot }}$, which is approximately the same under both power usage 
profiles, the performance gains in terms of power saving and energy efficiency are approximately the same.

\section{NumericAl RESUlts}

We present numerical results to illustrate the performance of the proposed admission control and power adaptation algorithms. The network setting is shown in Fig. 1 where there are 9 femtocells located at the edge of the central macrocell in a cluster of macrocells. The distance between two neighboring femtocells is $120 \mathrm{~m}$. We assume there are 2 classes of FUEs in each femtocell where class-one and class-two FUEs are located in the inner and outer circular regions whose radii from the corresponding FBS are $15 \mathrm{~m}$ and $30 \mathrm{~m}$, respectively. To calculate the required number of subchannels for FUEs of each class (i.e., $\underline{s}_{i, c}^{(f)}$ in $(7)$ ), we consider the worst case where FUEs are located on the boundary of the corresponding inner and outer regions (i.e., distances from these worst class-1 and class-2 FUEs to their FBS are $15 \mathrm{~m}$ and $30 \mathrm{~m}$, respectively). Moreover, we assume that MUEs will attempt to connect with a nearest FBS when they enter a circular area whose radius is $40 \mathrm{~m}$ from the underlying FBS. To calculate the rate requirements for MUEs in (8), we also assume the worst case where MUEs are located on the boundary of this circular area.

We calculate the long-term channel gains based on the corresponding distance. We assume that the distance $d_{i j}$ from BS $j$ to MUEs or FUEs associated with femtocell $i$ can be approximated by the distance from BS $j$ to FBS $i$. Also, the path-loss in $\mathrm{dB}$ corresponding to $d_{i j k}$ is calculated as [15]:

$$
\begin{aligned}
L\left(d_{i j k}\right) & =\left[44.9-6.55 \log _{10}\left(h_{B S}\right)\right] \log _{10}\left(d_{i j k}\right)+34.46 \\
& +5.83 \log _{10}\left(h_{B S}\right)+23 \log _{10}\left(f_{c} / 5\right)+n_{i j k} W_{i j k}
\end{aligned}
$$

where $h_{B S}$ is the BS height, which is chosen to be $25 \mathrm{~m}$ and $10 \mathrm{~m}$ for MBSs and FBSs, respectively (i.e., depending on whether BS $j$ in this loss calculation corresponds to MBSs or FBSs); $f_{c}$ is the carrier frequency in $\mathrm{GHz}$ which is set to $2 \mathrm{GHz}$. In addition, $W_{i j k}$ is the wall loss, and $n_{i j k}$ denotes the number of walls. For communications from an FBS to its associated FUEs, we choose $W_{i j k}=5 \mathrm{~dB}$ (i.e., indoor light walls) and $n_{i j k}=1$; for communications from an FBS to an indoor FUE connected with a different FBS, we assume $W_{i j k}=12 \mathrm{~dB}$ and $n_{i j k}=2$; for other cases we assume $W_{i j k}=12 \mathrm{~dB}$ and $n_{i j k}=1$. The path loss in the linear scale corresponding to $L\left(d_{i j k}\right)$ in $\mathrm{dB}$ is $10^{L\left(d_{i j k}\right) / 10}$.

Other system parameters are set as follows: FUEs' and MUEs' average service time $\mu_{i, c}^{(f)}=\mu_{i, c}^{(m)}=1$ minute, $\forall i, c$; arrival rates for all FUEs and MUEs are chosen to be the same (i.e., $\lambda_{i, c}^{(f)}=\lambda_{i, c}^{(m)}, \forall i, c$ ); maximum power of an MBS $P_{\mathrm{MBS}}^{\max }=80 \mathrm{~W}$; weighting factors in (29) are $w_{i, c}^{(m)}=w_{i, c}^{(f)}=1(\forall i, c)$; standard deviation of shadowing $\sigma=8 \mathrm{~dB}$; power scaling factor for Algorithm $1 \delta=0.9$; total number of available subchannels $N=6$; and parameter $K$ in step 16 of Algorithm 1 is chosen as $K=3$. Moreover, the minimum required rate for MUEs is $R_{\min }^{(m 1)} / W=6 \mathrm{~b} / \mathrm{s} / \mathrm{Hz}$; the minimum required rates for class- 1 and class-2 FUEs are $R_{\min }^{(f 1)} / W=10 \mathrm{~b} / \mathrm{s} / \mathrm{Hz}$ and $R_{\min }^{(f 2)} / W=4 \mathrm{~b} / \mathrm{s} / \mathrm{Hz}$, respectively. The target blocking probabilities used in Algorithm 1 are chosen as $P_{b}^{(m c)}=P_{b}^{(f c)}=0.05, \forall c$; target SINR of MUEs is $\gamma_{0}=3 \mathrm{~dB}$; and target outage probability for MUEs in (12) is

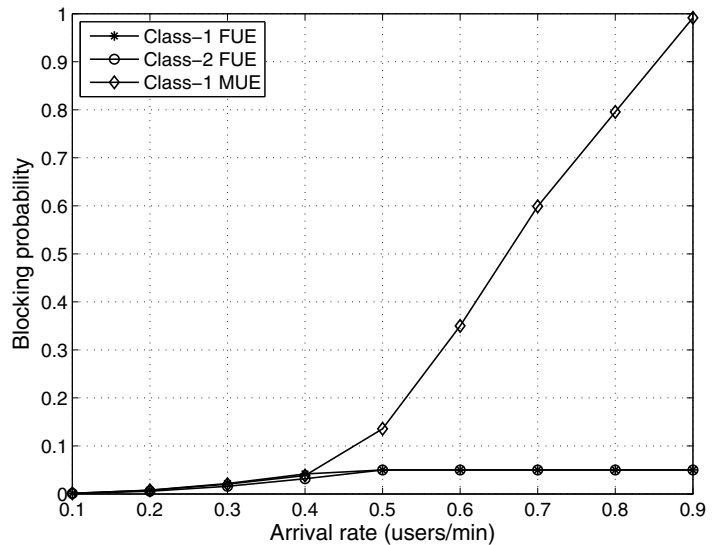

Fig. 4. Blocking probabilities in femtocell 1 when all FBSs use their maximum allowed power.

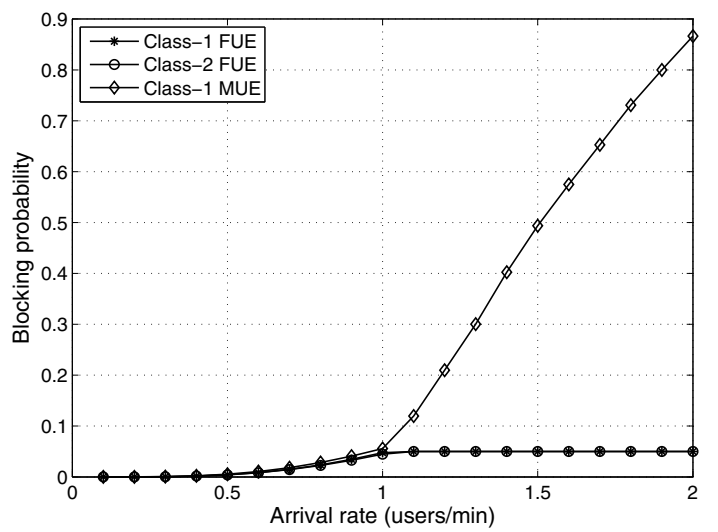

Fig. 5. Blocking probabilities in femtocell 2 when all FBSs use their maximum allowed power.

$P_{\mathrm{o}}^{(m)}=0.15$. We assume that the noise power and interference power from MBSs except the closest MBS of femtocell $i$ for SINR calculations in (1) and (2) are negligible compared to the interference powers from femtocells and the closest MBS. By using the techniques presented in Section III.C, the maximum allowable powers of each FBS for which we can still maintain the target outage probability of the MUEs $P_{\mathrm{o}}^{(m)}=0.15$ are $P_{\mathrm{FBS}}^{\max }=3.5 \mathrm{~mW}$ and $P_{\mathrm{FBS}}^{\max }=1.5 \mathrm{~mW}$ for the hybrid and closed access strategies, respectively.

Fig. 4 and Fig. 5 show the blocking probabilities achieved by the optimal admission control versus arrival rates in femtocells 1 and 2, respectively, as all FBSs use their maximum allowable transmission power under the hybrid access, which is equal to $P_{\mathrm{FBS}}^{\max }=3.5 \mathrm{~mW}$ to meet the target outage probability $P_{\circ}^{(m)}=0.15$. These figures confirm that the QoS requirements of FUEs in terms of blocking probabilities are always satisfied. As the network becomes congested, blocking probabilities of FUEs reach the target values (i.e., $P_{b}^{(f c)}=0.05$ ) while the blocking probability of MUEs increases slowly, and then it increases sharply before the system becomes unstable (i.e., it is not possible to support the target blocking probabilities of FUEs). In addition, the blocking probability of MUEs in Fig. 4 is much larger than that in Fig. 5 since users connecting 


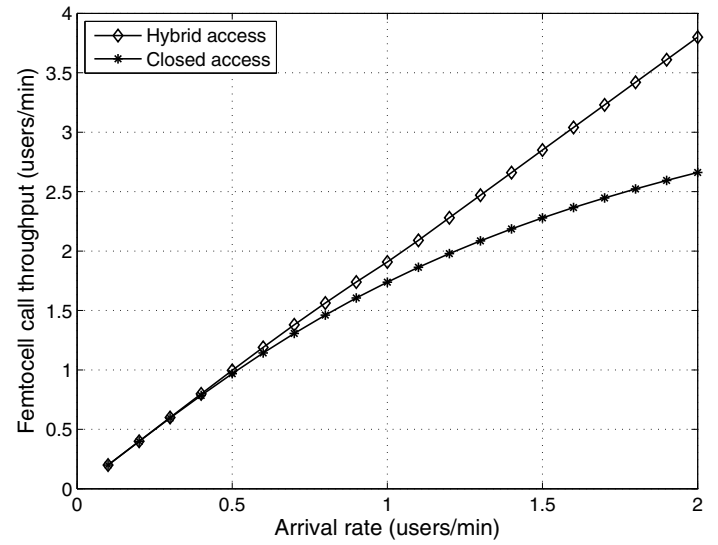

Fig. 6. Call throughput of femtocell 2 under the closed and proposed hybrid access strategies.

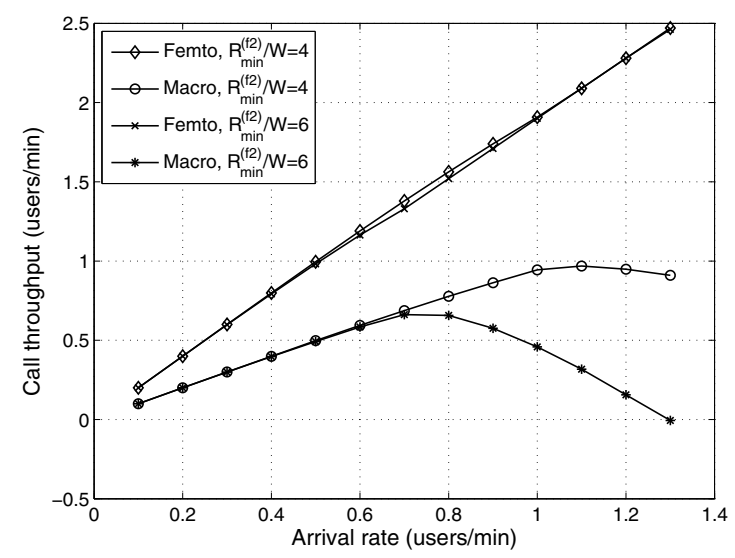

Fig. 7. Call throughput of FUEs and MUEs in femtocell 2 under different minimum rate constraints.

with FBS 1 receive relatively stronger interference from the nearest MBS compared to users connecting with FBS 2. This confirms the results in Proposition 3.

In Fig. 6, we plot the average call throughput achieved by all FUEs in femtocell 2 versus the arrival rate under the closed and proposed hybrid access strategies. This figure shows that the proposed hybrid access strategy achieves significantly higher call throughput than that due to the closed access strategy when the arrival rate is high. This performance gain comes from the fact that the maximum allowable transmission power of FBSs under the hybrid access is considerably larger than that under the closed access $\left(P_{\mathrm{FBS}}^{\max }=3.5 \mathrm{~mW}\right.$ and $P_{\mathrm{FBS}}^{\max }=1.5 \mathrm{~mW}$, respectively). Hence, even though FBSs must reserve some bandwidth for connecting MUEs under the hybrid access, the overall throughput achieved by FUEs is still higher than that under the closed access. The proposed hybrid access is, therefore, the win-win strategy for users of both network tiers.

To demonstrate the impacts of minimum rate requirements on the spectrum sharing between users of both tiers, we show the call throughputs achieved by FUEs and MUEs versus the arrival rate for femtocell 2 in Fig. 7 for $R_{\min }^{(f 2)} / W=4 \mathrm{~b} / \mathrm{s} / \mathrm{Hz}$ and $R_{\min }^{(f 2)} / W=6 \mathrm{~b} / \mathrm{s} / \mathrm{Hz}$ while rate requirements for other

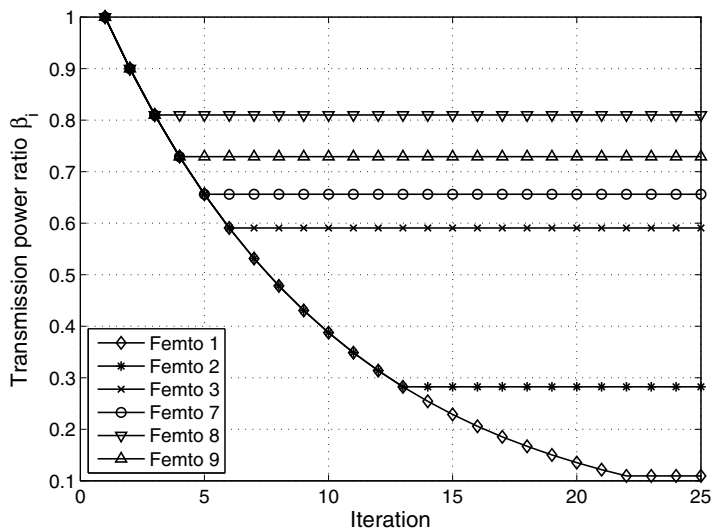

Fig. 8. Convergence of transmission power ratios under Algorithm 1.

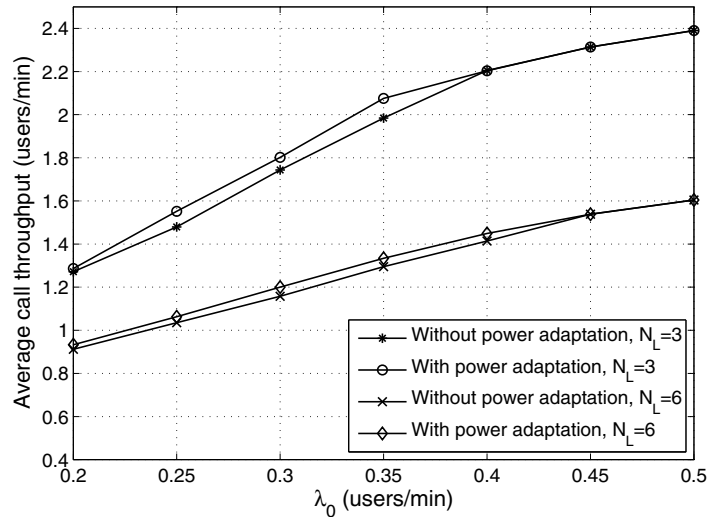

Fig. 9. Average throughput per femtocell with and without power adaptation.

user classes are $R_{\min }^{(m 1)} / W=6 \mathrm{~b} / \mathrm{s} / \mathrm{Hz}$ and $R_{\min }^{(f 1)} / W=10$ $\mathrm{b} / \mathrm{s} / \mathrm{Hz}$. As is evident from this figure, while the call throughput achieved by FUEs remains almost the same, the call throughput of MUEs becomes much smaller in the high arrival rate regime as $R_{\min }^{(f 2)} / W$ increases from $4 \mathrm{~b} / \mathrm{s} / \mathrm{Hz}$ to $6 \mathrm{~b} / \mathrm{s} / \mathrm{Hz}$. This means the proposed hybrid access strategy enables MUEs to appropriately exploit the radio resources beyond what is needed to support the required QoSs of FUEs.

We show the evolutions of the femtocell transmission power ratios $\beta_{i}$ for different femtocells under the synchronous power updates which demonstrate the convergence of Algorithm 1 in Fig. 8. There are $N_{L}=6$ lowly-loaded femtocells (femtocells 1 to 6) with $\lambda_{i, c}^{(f)}=\lambda_{i, c}^{(m)}=0.2$ users/min and the arrival rates of the remaining 3 highlyloaded femtocells are $\left[\lambda_{7, c}^{(f)}, \lambda_{8, c}^{(f)}, \lambda_{9, c}^{(f)}\right]=\left[\lambda_{0}, 3 \lambda_{0}, 4.5 \lambda_{0}\right]$ where $\lambda_{0}=0.3$ users $/ \mathrm{min}$. To keep the figure readable, we only show the transmission power ratios for 6 femtocells. In Fig. 9, we illustrate the average call throughput per femtocell achieved by the optimal admission control scheme with and without the power adaptation algorithm (i.e., Algorithm 1) where the arrival rates of $N_{L}$ lowly-loaded femtocells (femtocells 1 to $N_{L}$ ) are fixed at $\lambda_{i, c}^{(f)}=\lambda_{i, c}^{(m)}$ $=0.2 \mathrm{users} / \mathrm{min}$ and the arrival rates of the remaining highlyloaded femtocells are varied by using a parameter $\lambda_{0}$. Here, the arrival rates of highly-loaded femtocells are chosen as 


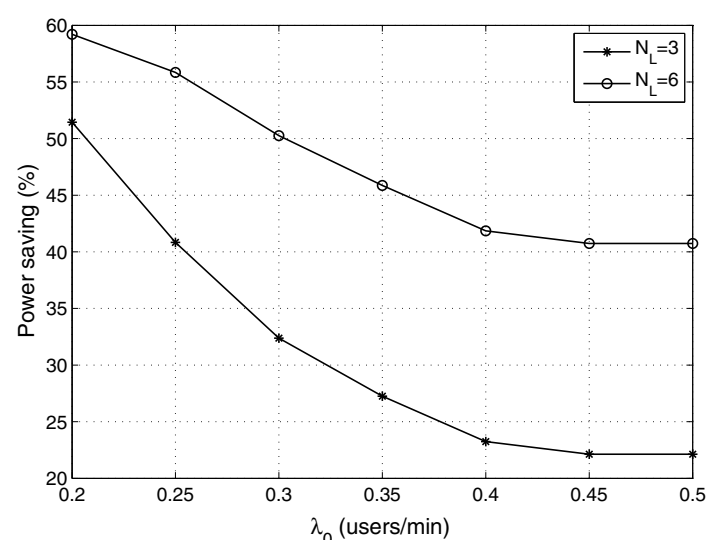

Fig. 10. Power saving due to femtocell power adaptation.

$\left[\lambda_{4, c}^{(f)}, \lambda_{5, c}^{(f)}, \ldots, \lambda_{9, c}^{(f)}\right]=\left[\lambda_{0}, 3 \lambda_{0}, 4.5 \lambda_{0}, \lambda_{0}, 3 \lambda_{0}, 4.5 \lambda_{0}\right]$ and $\left.\lambda_{4, c}^{(f)}, \lambda_{5, c}^{(f)}, \lambda_{6, c}^{(f)}\right]=\left[\lambda_{0}, 3 \lambda_{0}, 4.5 \lambda_{0}\right]$ for $N_{L}=3,6$, respectively. This figure shows that the proposed power adaptation algorithm can indeed maintain the average throughput for different values of arrival rates in the highly-loaded femtocells. In fact, Algorithm 1 slightly increases the average throughput for some values of arrival rates. The increase of throughput in highly-loaded femtocells is offset by slight decrease of throughput in other lightly-loaded femtocells. Therefore, the average throughput per femtocell remains almost the same for different values of arrival rates.

Finally, we present the power saving achieved by Algorithm 1 with the parameter $\lambda_{0}$ in Fig. 10 when the numbers of lowly-loaded femtocells are $N_{L}=3$ and 6. This figure confirms that significant power saving can be achieved by the proposed power adaptation algorithm when $\lambda_{0}$ is small, which are about $50 \%$ and $60 \%$ for $\lambda_{0}=0.2$ users $/ \mathrm{min}$ and $N_{L}=3$ and 6, respectively. Moreover, when the arrival rates of highlyloaded femtocells become larger, the power saving drops to about $22 \%$ and $41 \%$ for $N_{L}=3$ and $N_{L}=6$, respectively. In fact, the power saving for $N_{L}=6$ is larger than that for $N_{L}=3$ with high arrival rates because most of the power saving comes from the reduction in transmission powers of lightly-loaded femtocells.

\section{CONCLUSION}

We have considered the cross-layer resource allocation and admission control problem for OFDMA-based femtocell networks. Specifically, we have developed a unified model that captures co-tier and cross-tier interference as well as rate and blocking probability requirements for users of both tiers. Moreover, we have presented a novel distributed power adaptation algorithm, which has been proved to converge to the NE of the corresponding power adaptation game. Via numerical studies, we have demonstrated the desirable performance of the optimal admission control scheme in maintaining the QoS requirements for FUEs and optimally using the spectrum resources. Finally, it has been confirmed that a significant power saving can be achieved by the proposed joint admission control and power adaptation algorithm.

\section{APPENDIX A \\ SMDP-BASED ADMISSION CONTROL}

Given the system and action state spaces described in Section III.C, we are particularly interested in the admissible action space $A_{x}$ for a given system state $x$. In fact, $A_{x}$ comprises all possible actions that do not result in transition into a state that is not allowed (i.e., not in allowable state space $X$ in (16)). In addition, if $x=0$, then it is required that action $a=0$ is excluded from $A_{x}$ to prevent the system to be trapped in the zero state forever. Let $e_{i c}^{(f)}\left(e_{i c}^{(m)}\right)$ be a vector of dimension $C_{1}+C_{2}$, which is of the same size as that of the general state vector $x(t)$ having all zeros except the one at the same position of $x_{i c}^{(f)}\left(x_{i c}^{(m)}\right)$ in (15). Then, we can write $A_{x}$ as follows:

$$
\begin{aligned}
A_{x} \triangleq & \left\{a \in A: a_{i c}^{(f)}=0 \text { if } x+e_{i c}^{(f)} \notin X ;\right. \\
& \left.a_{i c}^{(m)}=0 \text { if } x+e_{i c}^{(m)} \notin X ; \text { and } a \neq 0 \text { if } x=0\right\} .
\end{aligned}
$$

We now analyze the dynamics of this SMDP, which is characterized by the state transition probabilities of the Markov chain obtained by embedding the system at arrival and departure instants. Specifically, we will determine transition probability $p_{x y}(a)$ from state $x$ to state $y$ when action $a$ is taken. Toward this end, let $\tau_{x}(a)$ denote the expected time until the next decision epoch after action $a$ is taken at system state $x$. Then, $\tau_{x}(a)$ can be calculated as the inverse of the cumulative arrival and departure rate with blocked arrivals taken into account. In particular, $\tau_{x}(a)$ can be calculated as follows [16], [33]:

$$
\begin{array}{r}
\tau_{x}(a)=\left[\sum_{c=1}^{C_{1}} \lambda_{i c}^{(f)} a_{i c}^{(f)}+\sum_{c=1}^{C_{1}} \mu_{i c}^{(f)} u_{i c}^{(f)}+\sum_{c=1}^{C_{2}} \lambda_{i c}^{(m)} a_{i c}^{(m)}\right. \\
\left.+\sum_{c=1}^{C_{2}} \mu_{i c}^{(m)} u_{i c}^{(m)}\right]^{-1}
\end{array}
$$

for $a \in A_{x}$. We are now ready to calculate transition probability $p_{x y}(a)$ of the underlying embedded Markov chain. This can be done by noting that the probability of a certain event (e.g., connection arrival and departure) is equal to the ratio between the rate of that event and the total cumulative event rate $1 / \tau_{x}(a)$. Therefore, the transition probability $p_{x y}(a)$ can be determined as follows:

$$
p_{x y}(a)= \begin{cases}\lambda_{i c}^{(f)} a_{i c}^{(f)} \tau_{x}(a), & \text { if } y=x+e_{i c}^{(f)} \\ \lambda_{i c}^{(m)} a_{i c}^{(m)} \tau_{x}(a), & \text { if } y=x+e_{i c}^{(m)} \\ \mu_{i c}^{(f)} u_{i c}^{(f)} \tau_{x}(a), & \text { if } y=x-e_{i c}^{(f)} \\ \mu_{i c}^{(m)} u_{i c}^{(m)} \tau_{x}(a), & \text { if } y=x-e_{i c}^{(m)} \\ 0, & \text { otherwise }\end{cases}
$$

where for simplicity we omit user index $i$ in both $\tau_{x}(a)$ and $p_{x y}(a)$. In the following, we formulate the optimal admission control problem using the above description of the underlying SMDP.

We formulate the admission control problem by defining a cost function as the weighted sum of blocking probabilities. In particular, we are interested in minimizing the following 
cost function:

$$
\begin{aligned}
\min _{z_{x a} \geq 0} & \sum_{c=1}^{C_{1}} w_{i c}^{(f)} \sum_{x \in X} \sum_{a \in A_{x}}\left(1-a_{i, c}^{(f)}\right) z_{x a} \tau_{x}(a) \\
+ & \sum_{c=1}^{C_{2}} w_{i c}^{(m)} \sum_{x \in X} \sum_{a \in A_{x}}\left(1-a_{i c}^{(m)}\right) z_{x a} \tau_{x}(a)
\end{aligned}
$$

where $z_{x a}$ denotes the rate of choosing action $a$ in state $x ; w_{i c}^{(m)}>0$ and $w_{i c}^{(f)}>0$ are weighting factors controlling the desired performance tradeoff. In this cost function, $B_{i c}^{(f)}=\sum_{x \in X} \sum_{a \in A_{x}}\left(1-a_{i c}^{(f)}\right) z_{x a} \tau_{x}(a)$ and $B_{i c}^{(m)}=$ $\sum_{x \in X} \sum_{a \in A_{x}}\left(1-a_{i c}^{(m)}\right) z_{x a} \tau_{x}(a)$ express the blocking probabilities of class- $c$ FUEs and MUEs, respectively, where $z_{x a} \tau_{x}(a)$ represents the probability that action $a$ is taken for a given system state $x$.

In addition, we impose blocking probability constraints for FUEs as follows:

$$
B_{i c}^{(f)}=\sum_{x \in X} \sum_{a \in A_{x}}\left(1-a_{i c}^{(f)}\right) z_{x a} \tau_{x}(a) \leq P_{b}^{(f c)}, 1 \leq c \leq C_{1} .
$$

Moreover, we impose other standard constraints for an MDP as follows [33]:

$$
\begin{aligned}
\sum_{a \in A_{y}} z_{y a}-\sum_{x \in X} \sum_{a \in A_{x}} p_{x y}(a) z_{x, a} & =0, \forall y \in X \\
\sum_{x \in X} \sum_{a \in A_{x}} z_{x a} \tau_{x}(a) & =1
\end{aligned}
$$

which describe the balance equation and the normalization condition, respectively [33]. The optimal admission control policy can be obtained as follows. We calculate optimal $z_{x a}^{*}$ by solving the linear program (29)-(32). Then, we can determine an optimal randomized admission control policy as follows: for each system state $x$ the probability of choosing action $a \in A_{x}$ can be calculated as $\theta_{x}(a)=z_{x a}^{*} \tau_{x}(a) / \sum_{a} z_{x a}^{*} \tau_{x}(a)$. These probabilities can be calculated offline and applied for online admission control for different system states. In the above model, we do not impose blocking probability constraints for MUEs. However, these additional constraints can be easily added to the model. Specifically, if $P_{b}^{(m c)}$ is the maximum tolerable blocking probability for class- $c$ MUEs, then additional blocking probability constraints can be written as

$$
\begin{aligned}
B_{i c}^{(m)}=\sum_{x \in X} \sum_{a \in A_{x}}\left(1-a_{i c}^{(m)}\right) z_{x a} \tau_{x}(a) & \leq P_{b}^{(m c)}, \\
1 & \leq c \leq C_{2} .
\end{aligned}
$$

Then, we can calculate optimal $z_{x a}^{*}$ by solving the linear program (29)-(32), (33), and find the optimal admission solution accordingly. This calculation is indeed used in Algorithm 1.

\section{REFERENCES}

[1] G. Boudreau, J. Panicker, N. Guo, R. Wang, and S. Vrzic, "Interference coordination and cancellation for $4 \mathrm{G}$ networks," IEEE Commun. Mag., vol. 44, no. 4, pp. 74-81, Apr. 2009.

[2] D. Lopez-Perez, A. Valcarce, G. de la Roche, and J. Zhang, "OFDMA femtocells: a roadmap on interference avoidance," IEEE Commun. Mag., vol. 47, no. 9, pp. 41-48, Sep. 2009.

[3] G. d. 1. Roche, A. Valcarce, D. Lopez-Perez, and J. Zhang, "Access control mechanisms for femtocells," IEEE Commun. Mag., vol. 48, no. 1, pp. 33-39, Jan. 2010.
[4] D. Lopez-Perez, A. Valcarce, A. Ladanyi, G. d. 1. Roche, and J. Zhang, "Intracell handover for interference and handover mitigation in OFDMA two-tier macrocell-femtocell networks," EURASIP J. Wireless Commun. Netw., 2010.

[5] I. Guvenc, M. R. Jeong, F. Watanabe, and H. Inamura, "A hybrid frequency assignment for femtocells and coverage area analysis for cochannel operation," IEEE Commun. Lett., vol. 12, no. 12, pp. 880882, Dec. 2008.

[6] D. T. Ngo, L. B. Le, and T. Le-Ngoc, "Distributed Pareto-optimal power control for utility maximization in femtocell networks," IEEE Trans. Wireless Commun., vol. 11, no. 10, pp. 3434-3446, Oct. 2012.

[7] D. T. Ngo, L. B. Le, T. Le-Ngoc, E. Hossain, and D. I. Kim, "Distributed interference management in two-tier CDMA femtocell networks," IEEE Trans. Wireless Commun., vol. 11, no. 3, pp. 979-989, Mar. 2012.

[8] V. Chandrasekhar and J. G. Andrews, "Spectrum allocation in tiered cellular networks," IEEE Trans. Commun., vol. 57, no. 10, pp. 30593068, Oct. 2009.

[9] P. Xia, V. Chandrasekhar, and J. G. Andrews, "Open vs. closed access femtocells in the uplink," IEEE Trans. Wireless Commun., vol. 9, no. 10, pp. 3798-3809, Dec. 2010.

[10] J.-H. Yun and K. G. Shin, "Adaptive interference management of OFDMA femtocells for co-channel deployment," IEEE J. Sel. Areas Commun., vol. 29, no. 6, pp. 1225-1241, June 2011.

[11] M.-S. Kim, H. W. Je, and F. A. Tobagi, "Cross-tier interference mitigation for two-tier OFDMA femtocell networks with limited macrocell information," in Proc. 2010 IEEE GLOBECOM.

[12] C.-H. Ko and H.-Y. Wei, "On-demand resource-sharing mechanism design in two-tier OFDMA femtocell networks," IEEE Trans. Veh. Technol., vol. 60, no. 3, pp. 1059-1071, Mar. 2011.

[13] M. E. Sahin, I. Guvenc, M.-R. Jeong, and H. Arslan, "Handling CCI and ICI in OFDMA femtocell networks through frequency scheduling," IEEE Trans. Consumer Electron., vol. 55, no. 4, pp. 1936-1944, Nov. 2009.

[14] S. Namal, K. Ghaboosi, M. Bennis, A. B. MacKenzie, and M. LatvaAho, "Joint admission control \& interference avoidance in selforganized femtocells," in Proc. 2010 Asilomar Conf. Signals, Syst. Comput.

[15] M. Rahman and H. Yanikomeroglu, "Enhancing cell-edge performance: a downlink dynamic interference avoidance scheme with inter-cell coordination," IEEE Trans. Wireless Commun., vol. 9, no. 4, pp. 14141425, Apr. 2010.

[16] J. Choi, T. Kwon, Y. Choi, and M. Naghshineh, "Call admission control for multimedia services in mobile cellular networks: a Markov decision approach," in Proc. 2000 IEEE Int. Symp. Comput. Commun.

[17] S. Singh, V. Krishnamurthy, and H. V. Poor, "Integrated voice/data cal admission control for wireless DS-CDMA systems," IEEE Trans. Signal Process., vol. 50, no. 6, pp. 1483-1495, June 2002.

[18] P. Viswanath, D. Tse, and R. Laroia, "Opportunistic beamforming using dumb antennas," IEEE Trans. Inf. Theory, vol. 48, no. 6, pp. 1277-1294, June 2002.

[19] A. M. D. Turkmani, "Probability of error for M-branch macroscopic selection diversity," IEE Proc. I Commun. Speech Vision, vol. 139, no. 1, pp. 71-78, Feb. 1992.

[20] H. Fu and D. I. Kim, "Analysis of throughput and fairness with downlink scheduling in WCDMA networks," IEEE Trans. Wireless Commun., vol. 5, no. 8, pp. 2164-2174, Aug. 2006.

[21] D. I. Kim, E. Hossain, and V. K. Bhargava, "Downlink joint rate and power allocation in cellular multi-rate WCDMA systems," IEEE Trans. Wireless Commun., vol. 2, pp. 69-80, Jan. 2003.

[22] C. J. Chen and L. C. Wang, "A unified capacity analysis for wireless systems with joint multiuser scheduling and antenna diversity in Nakagami fading channels," IEEE Trans. Wireless Commun., vol. 54, no. 3, pp. 469-478, Mar. 2006.

[23] N. B. Mehta, J. Wu, A. F. Molisch, and J. Zhang, "Approximating a sum of random variables with a lognormal," IEEE Trans. Wireless Commun., vol. 6, no. 7, pp. 2690-2699, July 2007.

[24] G. Miao, N. Himayat, G. Y. Li, and S. Talwar, "Distributed interferenceaware energy-efficient power optimization," IEEE Trans. Wireless Commun., vol. 10, no. 4, pp. 1323-1333, Apr. 2011.

[25] M. C. Gursoy, D. Qiao, and S. Velipasalar, "Analysis of energy efficiency in fading channels under QoS constraints," IEEE Trans. Wireless Commun., vol. 8, no. 8, pp. 4252-4263, Aug. 2009.

[26] D. Qiao, M. C. Gursoy, and S. Velipasalar, "Energy efficiency in the low-SINR regime under queueing constraints and channel uncertainty," IEEE Trans. Wireless Commun., vol. 59, no. 7, pp. 2006-2017, July 2011.

[27] X. Chu, Y. Wu, D. Lopez-Perez, and X. Tao, "On providing downlink services in collocated spectrum-sharing macro and femto networks," 
IEEE Trans. Wireless Commun., vol. 10, no. 12, pp. 4306-4315, Dec. 2011.

[28] W. C. Chung, T. Q. S. Quek, and M. Kountouris, "Throughput optimization, spectrum allocation, and access control in two-tier femtocell networks," IEEE J. Sel. Areas Commun., vol. 30, no. 3, pp. 561-574, Apr. 2012.

[29] Z. Q. Luo and S. Zhang, "Dynamic spectrum management: complexity and duality," IEEE J. Sel. Topics Signal Process., vol. 2, no. 1, pp. 57-73, Feb. 2008.

[30] 3GPP TR V0.0.9, “Overview of 3GPP Release 11,” Jan. 2012. Available: http://www.3gpp.org

[31] Z. Niu, "TANGO: traffic-aware network planning and green operation," IEEE Wireless Commun., vol. 18, no. 5, pp. 25-29, 2011.

[32] G. L. Stuber, Principles of Mobile Communication. Kluwer Academic, 1996.

[33] M. Puterman, Markov Decision Processes: Discrete Stochastic Dynamic Programming. Wiley, 1994.

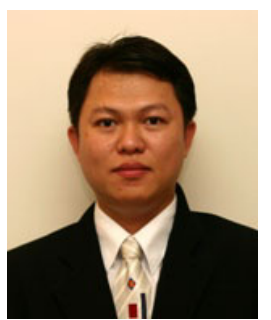

Long Bao Le (S'04-M'07-SM'12) received the B.Eng. (with Highest Distinction) degree from Ho Chi Minh City University of Technology, Vietnam, in 1999, the M.Eng. degree from Asian Institute of Technology, Pathumthani, Thailand, in 2002, and the Ph.D. degree from the University of Manitoba, Winnipeg, MB, Canada, in 2007.

From 2008 to 2010, he was a postdoctoral research associate with Massachusetts Institute of Technology, Cambridge, MA. Since 2010, he has been an assistant professor with the Institut National de la Recherche Scientifique (INRS), Université du Québec, Montreal, QC, Canada, where he leads a research group working on cognitive radio and dynamic spectrum sharing, radio resource management, network control and optimization.

Dr. Le is a member of the editorial board of IEEE COMMUNICATIONS SURVEYS AND TUTORIALS and IEEE WIRELESS COMMUNICATIONS LETTERS. He has served as technical program committee co-chairs of the Wireless Networks track at IEEE VTC2011-Fall and the Cognitive Radio and Spectrum Management track at IEEE PIMRC2011. He is a Senior Member of the IEEE.

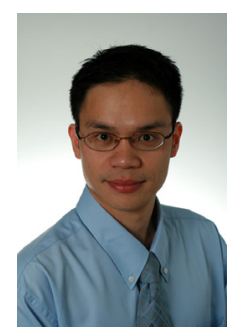

Dusit Niyato (M'09) received the BE degree from King Mongkuts Institute of Technology Ladkrabang (KMITL), Bangkok, Thailand in 1999, and the PhD degree in electrical and computer engineering from the University of Manitoba, Canada, in 2008. He is currently an assistant professor in the School of Computer Engineering at the Nanyang Technological University, Singapore. His research interests are in the area of radio resource management in cognitive radio networks and broadband wireless access networks. He is a member of the IEEE.

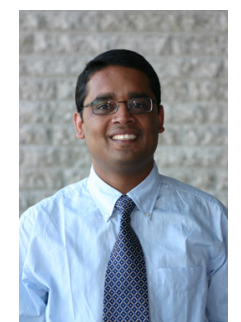

Ekram Hossain (S'98-M'01-SM'06) is a Full Professor in the Department of Electrical and Computer Engineering at University of Manitoba, Winnipeg, Canada. He received his Ph.D. in Electrical Engineering from University of Victoria, Canada, in 2001. Dr. Hossain's current research interests include design, analysis, and optimization of wireless/mobile communications networks, $\operatorname{cog}$ nitive radio systems, and network economics. He has authored/edited several books in these areas (http://www.ee.umanitoba.ca/ ekram). Dr. Hossain serves as the Editor-in-Chief for the IEEE COMMUNICATIONS SURVEYS AND TUTORIALS (for the term 2012-2013) and an Editor for IEEE Wireless Communications. Previously, he served as the Area Editor for the IEEE TRANSACTIONS ON WIRELESS COMMUNICATIONS in the area of "Resource Management and Multiple Access" from 2009-2011 and an Editor for the IEEE Transactions on Mobile Computing from 2007-2012. Dr. Hossain has won several research awards including the University of Manitoba Merit Award in 2010 (for Research and Scholarly Activities), the 2011 IEEE Communications Society Fred Ellersick Prize Paper Award, and the IEEE Wireless Communications and Networking Conference 2012 (WCNC'12) Best Paper Award. He is a registered Professional Engineer in the province of Manitoba, Canada.

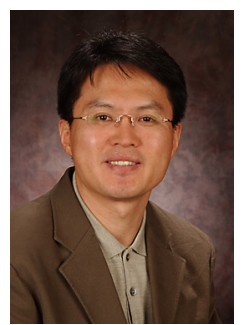

Dong In Kim (S'89-M'91-SM'02) received the $\mathrm{Ph} . \mathrm{D}$. degree in electrical engineering from the University of Southern California, Los Angeles, in 1990.

He was a tenured Professor with the School of Engineering Science, Simon Fraser University, Burnaby, BC, Canada. Since 2007, he has been with Sungkyunkwan University (SKKU), Suwon, Korea, where he is currently a Professor with the School of Information and Communication Engineering. His research interests include wireless cellular, relay networks, and cross-layer design.

Dr. Kim has served as an Editor and a Founding Area Editor of CrossLayer Design and Optimization for the IEEE TRANSACTIONS ON WIRELESS Communications from 2002 to 2011 . From 2008 to 2011, he served as the Co-Editor-in-Chief for the Journal of Communications and Networks. He is currently an Editor of Spread Spectrum Transmission and Access for the IEEE TRANSACTIONS ON COMmuniCATIONS and the Founding Editor-inChief for the IEEE WiRELESS COMMUNICATIONS LETTERS.

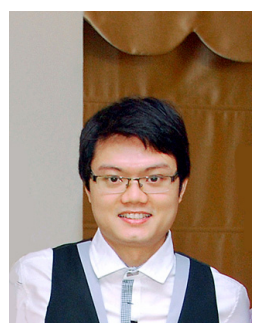

Dinh Thai Hoang received his Bachelor degree in Electronics and Telecommunications from Hanoi University of Technology (HUT), Vietnam in 2009. From 2010 to 2012 he worked as a research assistant at Nanyang Technological University (NTU), Singapore. He has received the NTU research scholarship and he is currently working toward his Ph.D. degree in School of Computer Engineering, NTU under Professor Dusit Niyato's supervision. His research interests include cooperative networked systems, and resource allocation for wired and wireless networks. 